\title{
WFPC2 Images of the Central Regions of Early-Type Galaxies - I. The Data
}

\author{
Armin Rest, Frank C. van den Bosch ${ }^{1,2}$ \\ Department of Astronomy, University of Washington, Seattle, WA 98195, USA \\ Walter Jaffe \\ Leiden Observatory, P.O. Box 9513, 2300 RA Leiden, The Netherlands \\ Hien Tran, Zlatan Tsvetanov, Holland C. Ford, James Davies, Joanna Schafer \\ Department of Physics \& Astronomy, Johns Hopkins University, Baltimore, MD 21218, USA
}

\footnotetext{
${ }^{1}$ Hubble Fellow

${ }^{2}$ Current Address: Max-Planck Institut für Astrophysik, Karl-Schwarzschild Strasse 1, Postfach 1317,85741 Garching, Germany
} 


\begin{abstract}
We present high resolution $R$-band images of the central regions of 67 early-type galaxies obtained with the Wide Field and Planetary Camera 2 (WFPC2) aboard the Hubble Space Telescope (HST). This homogeneously selected sample roughly doubles the number of early-type galaxies that have now been imaged at HST resolution, and complements similar data on the central regions of radio galaxies and the bulges of spiral galaxies. Our sample strikingly confirms the complex morphologies of the central regions of early-type galaxies which have become apparent from previous studies with HST. In particular, we detect dust, either in the form of nuclear disks or with a filamentary distribution, in 43 percent of all galaxies, in good agreement with previous estimates. In addition, we find evidence for embedded stellar disks in a remarkably large fraction of 51 percent. In 14 of those galaxies the disk-like structures are misaligned with the main galaxy, suggesting that they correspond to stellar bars in $\mathrm{S} 0$ galaxies. We analyze the luminosity profiles of the galaxies in our sample, and classify galaxies according to their central cusp slope. To a large extent we confirm the results from previous HST surveys in that early-type galaxies reveal a clear dichotomy: the bright ellipticals $\left(M_{B} \lesssim-20.5\right)$ are generally boxy and have luminosity profiles that break from steep outer power-laws to shallow inner cusps (referred to as 'core' galaxies). The fainter ellipticals, on the other hand, typically have disky isophotes and luminosity profiles that lack a clear break and have a steep central cusp (referred to as 'power-law' galaxies). The advantages and shortcomings of classification schemes utilizing the extrapolated central cusp slope $\gamma$ are discussed, and it is shown that $\gamma$ might be an inadequate representation for galaxies whose luminosity profile slope changes smoothly with radius rather than resembling a broken power-law. Thus we introduce a new, alternative parameter, and show how this affects the classification. In fact, we find evidence for an 'intermediate' class of galaxies, that cannot unambiguously be classified as either core or power-law galaxies, and which have central cusp slopes and absolute magnitudes intermediate between those of core and power-law galaxies. It is unclear at present, however, whether these galaxies make up a physically distinct class or whether distance and/or resolution effects cause them to loose their distinct core or power-law characteristics.
\end{abstract}

Subject headings: galaxies: elliptical and lenticular, $\mathrm{cD}$ — galaxies: nuclei — galaxies: structure. 


\section{Introduction}

Ever since the seminal study by Davies et al. (1983) it is known that elliptical galaxies can be divided in two distinct classes. The bright ellipticals $\left(M_{B} \lesssim-20.5\right)$ generally have a boxy appearance, are pressure supported, and often show some form of radio and/or X-ray activity. Low luminosity ellipticals, on the other hand, often have disky isophotes, are rotationally supported, and generally lack significant amounts of activity (Bender 1988; Bender et al. 1989; Nieto et al. 1988). Before the Hubble Space Telescope (HST) came online, very little was known about the nuclear properties of these systems. Based on ground-based observations, with a typical seeing of $\gtrsim 1$ arcsec, ellipticals were considered to have constant density cores with a size that correlates with global galaxy properties (e.g., Lauer 1985; Kormendy 1985). High-resolution imaging surveys with the HST, however, revealed that the central brightness profiles of early-type galaxies are well approximated by a power-law, $I(r) \propto r^{-\gamma}$ with $\gamma>0$ (e.g., Lauer et al. 1991, 1992; Crane et al. 1993; Ferrarese et al. 1994; Kormendy et al. 1994; Lauer et al. 1995; Carollo et al. 1997a). Furthermore, the distribution of $\gamma$ was found to be bimodal and to follow the dichotomy of global properties inferred from ground-based observations (Jaffe et al. 1994; Lauer et al. 1995; Faber et al. 1997) and to be correlated with the global X-ray emission (Pellegrini 1999). Bright ellipticals have luminosity profiles that are well fit by a double power-law with a break radius of typically a few hundred parsecs. The inner power-law slope has values of $0<\gamma \lesssim 0.3$. This contrasts strongly with the low-luminosity ellipticals whose luminosity profiles lack a clear break radius and have central power-law profiles with $\gamma \sim 0.8$ on average (Jaffe et al. 1994; Ferrarese et al. 1994; Lauer et al. 1995; Byun et al. 1996; Gebhardt et al. 1996; Faber et al. 1997; Quillen, Bower \& Stritzinger 2000). Following the nomenclature of Lauer et al. (1995) we refer to the former as core galaxies and to the latter as power-law galaxies.

In addition to the central density cusps, the images from the HST have revealed that a large fraction of early-type galaxies harbor significant amounts of dust $\left(\sim 10^{3}-10^{7} \mathrm{M}_{\odot}\right)$, either in the form of a nuclear disk, or of a more complex, filamentary or patchy morphology (Jaffe et al. 1994; Lauer et al. 1995; van Dokkum \& Franx 1995). In several cases, nuclear stellar disks with scale-lengths as small as $\sim 20$ pc, are found (e.g., van den Bosch et al. 1994; van den Bosch, Jaffe \& van der Marel 1998; Scorza \& van den Bosch 1998). Both the gaseous and stellar disks have been shown to be powerful tools for determining the central densities of their parent galaxies, and for detecting massive black holes (Harms et al. 1994; Ferrarese, Ford \& Jaffe 1996; van den Bosch \& de Zeeuw 1996; Macchetto et al. 1997; Bower et al. 1998; Cretton \& van den Bosch 1999; van der Marel \& van den Bosch 1999). Mainly because of these nuclear disk structures the number of galaxies with solid detections of massive black holes (BHs) has increased strongly over the past years, and we have reached the point where we can start to address the demographics of BHs (see Kormendy \& Richstone 1995, Ford et al. 1998, Ho 1999, van der Marel 1999a, and Macchetto 1999 for recent reviews).

Black holes also play an important role in connection with the observed central cusps. Two different scenarios have been proposed that rely on the presence of massive black holes to explain the correlation between cusp slope and luminosity. Faber et al. (1997) have suggested that luminous galaxies are the result of mergers, whereby BHs in the progenitors sink to the center and 
excavate a core (see also Begelman, Blandford \& Rees 1980; Makino \& Ebisuzaki 1996; Quinlan 1996; Quinlan \& Hernquist 1997). Van der Marel (1999b), on the other hand, starts with the assumption of constant density cores, and shows that the adiabatic growth of central BHs can create cusps whose slopes correlate with luminosity as observed. Thus, whereas the origin of the relation between cusp slope, luminosity and black hole mass is still unknown, it has become apparent that massive black holes are omnipresent in galactic nuclei.

Despite the enormous wealth of new data, we are still far from a proper understanding of the structure and formation of the central regions of early-type galaxies. Not only have the new HST observations of these systems changed our view of their central regions, they have also raised several new problems:

- Is the bimodality in cusp slopes real? Data on elliptical galaxies with kinematically distinct cores seems to suggest a much more continuous transition (Carollo et al. 1997a).

- What is the origin of the correlation between cusp slope and global characteristics of early-type galaxies?

- What fraction of early-type galaxies contain nuclear disks of stars or dust/gas, and how are these structures related to other properties of their parent galaxies?

- Is there a relation between the central structure of galaxies and the presence or absence of nuclear activity?

- How does the mass of a central BH correlate with other properties as bulge mass and cusp slope, and what role do BHs play in determining the properties of the central regions of galaxies?

In order to address these questions one needs a large and unbiased sample of early-type galaxies that have been imaged at comparable resolution. On the order of 80 early-type galaxies have been imaged with the HST, most of them before the refurbishment mission. We have obtained HST images with WFPC2 of the central regions of 67 early-type galaxies as part of our HST snap shot program \# 6357. This roughly doubles the total number of early-type galaxies that have been imaged with the HST. Together with the data taken so far and with similar samples of spiral bulges (Phillips et al. 1996; Carollo et al. 1997b; Carollo, Stiavelli \& Mack 1998) and radio galaxies (de Koff et al. 1996; McCarthy et al. 1997; Martel et al. 1999; Verdoes Kleijn et al. 1999) these images provide a data base for the investigation of correlations of properties such as dust, metallicity, colors, activity, and nuclear structure.

In this paper, the first in a series, we present the data and describe the luminosity profiles and isophotal structures. More detailed analyses of the dust, stellar disks, and the central parameter relations are deferred to future papers. 


\section{The Sample}

The sample is compiled from the Lyon/Meudon Extragalactic Database (LEDA) by selecting all early-type galaxies with radial velocities less than $3400 \mathrm{~km} \mathrm{~s}^{-1}$, an absolute $V$-band magnitude less than -18.5 , and absolute galactic latitude exceeding 20 degrees (to minimize the effects of galactic extinction). In total 130 galaxies made it into the sample, of which 68 have been observed successfully by HST. Thus, although our sample is by no means complete, it has been homogeneously selected. One galaxy, UGC 5467, turned out to be a spiral galaxy and will be discarded in what follows. Note that, although we have attempted to discard obvious duplicates (WFPC2) from our sample, several of our objects have been observed in other HST programs since the time our sample was selected. In most cases, however, a different filter was used than for the data presented here.

Global properties of the 67 galaxies in our sample, taken from the LEDA (see Paturel et al. 1997), are listed in Table 1. Absolute magnitudes (column 2) are based on the distances listed in column 4, and based on the Virgo-centric infall corrected heliocentric velocities assuming $H_{0}=80 \mathrm{~km} \mathrm{~s}^{-1} \mathrm{Mpc}^{-1}$ (see Paturel et al. 1997 for details). In addition, we list the $1.4 \mathrm{GHz}$ radio fluxes, taken from the NRAO VLA Sky Survey (NVSS; Cordon et al. 1998), as well as a far-infrared magnitudes defined as

$$
m_{\mathrm{FIR}}=-2.5 \log \left(2.58 f_{60}+f_{100}\right)+14.75
$$

(cf. de Vaucouleurs et al. 1991). Here $f_{60}$ and $f_{100}$ are the q60 $\mu \mathrm{m}$ and $100 \mu \mathrm{m}$ IRAS fluxes measured by one of us, adopting a similar approach to that taken by Knapp et al. (1989).

\section{Observations and Data Reduction}

\subsection{Observing strategy}

We used the WFPC2 to obtain broad band images of the galaxies in our sample, using the F702W filter. This filter, centered on $\lambda=6997 \AA$ and with a FWHM of $\sim 1481 \AA$, was chosen to compromise between maximizing the number of photons and minimizing the effects of dust on the observed morphologies. The nuclei of the galaxies were centered on the Planetary Camera CCD (PC), which consists of $800 \times 800$ pixels of $0.046^{\prime \prime} \times 0.046^{\prime \prime}$ each. All exposures were taken with the telescope guiding in fine lock, yielding a RMS telescope jitter of $\sim 3$ milli-arcseconds. More detailed information on the WFPC2 can be found in Biretta et al. (1996).

Previous HST/WFPC2 observations of ellipticals have shown that the power-law galaxies have typically a central surface brightness in the $V$-band of $\mu_{0} \approx 14.5$, whereas core-galaxies typically have $\mu_{0} \approx 16.5$. Our aim is to obtain a signal-to-noise $(\mathrm{S} / \mathrm{N})$ of 20 or larger in the center of the galaxies. This prompted us to use a total exposure time of 1000 seconds with the analogue-to-digital gain set to 13.99 electrons/DN (DN is the number of counts). With this

\footnotetext{
${ }^{3}$ www-obs.univ-lyon1.fr
} 
setting the read-out noise is 7.02 electrons. To facilitate cosmic ray removal, and to guard against saturation in the presence of a bright nuclear point source, each exposure was split in two separate exposures of 300 and 700 seconds respectively.

\subsection{Data reduction}

The images are calibrated by the HST calibration 'pipeline' maintained by the Space Telescope Science Institute. The standard reduction steps include bias subtraction, dark current subtraction and flat-fielding, and are described in detail by Holtzman et al. (1995a).

Subsequent reduction is done using standard IRAF tasks. Using the data quality files and the WFIXUP task, bad pixels are corrected by means of a linear one-dimensional interpolation. The alignment between the 300 and 700 exposures is determined by comparing the isophote centers from initial analyses of the separate exposures. If the misalignment is significant the 300 sec exposure is shifted appropriately, using linear interpolation. A difference-image of the $700 \mathrm{sec}$ and (shifted) $300 \mathrm{sec}$ images is used to check the accuracy of the alignment. Next, the two images are combined with simultaneous removal of cosmic rays, using the IRAF task IMCOMBINE. The cosmic ray removal and bad pixel correction is checked by inspection of the residual images between the cleaned, combined image and each of the two original frames. Sky levels are determined from empty areas in the WF images, and accordingly subtracted. The sky-level is found to be small, typically $\sim 1 \mathrm{DN}$. The count rates in the broad-band F702W images are calibrated to magnitudes in the Landoldt $R$-band, as described in Holtzman et al. (1995b), and assuming a $V$ - $R$ color of 0.68. The magnitudes thus derived are checked using the inverse sensitivity and the zero point of the magnitude scale provided by the HST pipeline, whereby it is assumed that ellipticals have the spectrum of a K-III giant. The consistency is found to be better than 0.01 dex.

The 300 and 700 sec exposures are reduced separately as well. Cosmic rays are removed using the IRAF task COSMICRAY. These images are used to check the consistency of structures, for obtaining estimates of the uncertainties due to random noise, and for cases where saturation has affected the $700 \mathrm{sec}$ exposure (see $\S 5.2$ ).

Despite the high spatial resolution of the refurbished HST, the central few tenths of an arcsec of the galaxies are influenced by the effects of point-spread function (PSF) smearing. Because of the stability of the HST PSF, these effects can be corrected for by means of deconvolution with the Richardson-Lucy (RL) algorithm (Lucy 1974). Based on tests with model galaxies (see $\S 4.1$ below) we deconvolve the reduced $300 \mathrm{sec}, 700 \mathrm{sec}$ and combined images of each galaxy with 40 iterations of the RL algorithm. For three galaxies with faint and shallow surface brightness profiles (NGC 3613, NGC 4168 and NGC 4365), the RL-algorithm with 40 iterations ends up amplifying the noise too strongly for an accurate analysis of the central surface brightness profile, and only 15 iterations are used in these cases. For each galaxy we use a separate model PSF $\left(5.7^{\prime \prime} \times 5.7^{\prime \prime}\right)$, created with the TINYTIM software package (Krist 1992), centered on the location of the galaxy's 
center in the PC CCDf. Contour plots of the deconvolved images are presented in the Appendix.

\section{Data Analysis}

The main goal of this paper is to analyze the isophotal structure and luminosity profiles of the galaxies. For the isophotal analysis we use the isophote fitting task ELLIPSE in IRAF. For each isophote, the center, ellipticity $(\epsilon)$, and position angle $(\theta)$ are computed. In addition, the third and fourth order deviations of the isophote from a pure ellipse are determined. These are described by the amplitudes $\left(a_{n}\right.$ and $b_{n}$ with $\left.n=3,4\right)$ of the sin and $\cos 3 \theta$ and $4 \theta$ terms of the following Fourier expansion (see Jedrzejewski 1987):

$$
\frac{\delta r(\theta)}{r(\theta)}=\sum_{n=3}^{4}\left[a_{n} \sin (n \theta)+b_{n} \cos (n \theta)\right]
$$

For a perfectly elliptical isophote these coefficients are all equal to zero. Typically the absolute values of $a_{n}$ and $b_{n}$ are small, rarely exceeding 0.02 . The $b_{4}$ coefficient is of special interest, since positive (negative) $b_{4}$ values correspond to disky (boxy) isophotes.

In order to parameterize the luminosity profiles they are fitted with a double power law, known as the "Nuker"-law profile (Lauer et al. 1995):

$$
I(r)=I_{b} 2^{(\beta-\gamma) / \alpha}\left(\frac{r}{r_{b}}\right)^{-\gamma}\left[1+\left(\frac{r}{r_{b}}\right)^{\alpha}\right]^{(\gamma-\beta) / \alpha}
$$

which has $I(r) \propto r^{-\beta}$ at $r^{\alpha} \gg r_{b}^{\alpha}$, and $I(r) \propto r^{-\gamma}$ at $r^{\alpha} \ll r_{b}^{\alpha}$. The parameter $\alpha$ describes the sharpness of the break from the outer to the inner power law. The break-radius, $r_{b}$, is the radius of maximum curvature in log-log coordinates, and $I_{b}$ is the surface brightness at $r=r_{b}$. The Nuker law has been shown to accurately fit the central luminosity profiles of early-type galaxies (e.g., Byun et al. 1996). Note that equation (3) is only intended to fit the luminosity profiles in the inner $\sim 20^{\prime \prime}$ of early-type galaxies. For typical fitted values of $\beta$ there must be a further downturn in the profile at larger radii for the total luminosity to be finite.

\subsection{Model galaxies}

In order to test the accuracy of our reduction and analysis we constructed a large set of model galaxies. Each model galaxy has a luminosity profile of the form of equation (3), has constant ellipticity and position angle, and has perfectly elliptical isophotes (i.e., $a_{n}=b_{n}=0$ for $n \geq 3$ ). A fake image is created by integrating the model surface brightness distribution over each pixel, and by adding photon and read-out noise. Pixel sizes are taken the same as for the true data. The frame size of $200 \times 200$ pixels is smaller than the actual HST images, but satisfies the requirements for our testing purposes.

\footnotetext{
${ }^{4}$ The exact shape of the HST PSF depends on its location in the focal plane.
} 
The resulting image, refered to as 'unconvolved' image, is convolved with the HST PSF (the 'convolved' image), and subsequently deconvolved using $N_{\text {iter }}$ iterations of the RL algorithm (the 'deconvolved' image). The PSFs used for the convolution and deconvolution are constructed with TINYTIM, and differ slightly from each other in order to mimick errors in the exact PSF shape. The unconvolved and deconvolved model images are analyzed in the same way as the data: an isophotal analysis is performed, and we fit the Nuker-law profile to the major axis luminosity profile determined from the isophote fitting. The deconvolution is tested with 10, 15, 25, 40 and 80 iterations. The best results were obtained for $N_{\text {iter }}=40$, in good agreement with Lauer et al. (1998). However, for galaxies with shallow central surface brightness profiles $N_{\text {iter }}=15$ already suffices to accurately recover the unconvolved image, and more iterations only amplify the noise.

In total we have constructed $15 \times 50$ model galaxies. Each of the 15 sets of model galaxies is characterized by a value of $\gamma$, ranging from $\gamma=0$ to $\gamma=1.4$ in steps of 0.1 . For each set 50 model galaxies are created whose luminosity profile parameters are varied at the few percent level. The ellipticity and orientation of each model galaxy are chosen randomly from the intervals $[0,0.5]$ and $[-90,90]$, respectively. In addition, the center of the galaxy, in fractional pixels, is drawn randomly as well.

In Figure 1 we plot the isophotal parameters of one of the model galaxies with $\gamma=0.7$. Even though this model intrinsically has $\epsilon=0.26$ over its entire radial range, the ellipticities found by the isophote-fitting routine are significantly lower for $r \lesssim 0.2^{\prime \prime}$, even for the unconvolved image (solid triangles). In addition, the higher-order parameters become noisy and reveal fluctuations inside this radius. These effects owe mainly to the discrete pixel sampling and the sub-pixel interpolation used by the isophote-fitting routine, and indicate that one can not trust the isophotal parameters for $r \lesssim 0.2^{\prime \prime}(\sim 4$ pixels $)$. Note, however, that the isophotal parameters of the deconvolved model galaxy are remarkably similar to those of the unconvolved model, indicating that the deconvolution routine used does not introduce spurious artefacts.

In order to examine the accuracy with which we can recover the Nuker parameters of the luminosity profile used as input for the model, we first focus on the unconvolved images. For each model $i$ we compute

$$
\Delta \mu_{i}(r)=\mu_{0, i}(r)-\mu_{\text {iso }, i}(r)
$$

with $\mu_{0, i}(r)$ the input surface brightness profile (in magn. $\operatorname{arcsec}^{-2}$ ) of equation (3), and $\mu_{\text {iso }, i}(r)$ the major axis lumninosity profile determined from the isophotal analysis of the unconvolved image. For each of the 15 sets of model galaxies (that have the same value of $\gamma$ ), we then compute the average $\langle\Delta \mu\rangle(r)$ and the corresponding standard deviation $\sigma_{\Delta \mu}(r)$, where the average is over the 50 models in each set.

Figure 2 plots $\langle\Delta \mu\rangle(r)$ and $\sigma_{\Delta \mu}(r)$ for 8 of the 15 sets. For $r \gtrsim 0.2^{\prime \prime}$ the luminosity profiles derived from the isophotal analysis are in excellent agreement with the intrinsic profile. For smaller radii, however, the error increases strongly, especially for the profiles with steep central cusp slopes. Even more worrysome is that the average residual $\langle\Delta \mu\rangle(r)$ strongly deviates from zero at small radii, implying that the main contribution to the error is of systematic rather than random nature. This is a reflection of the problems with the isophotal analysis due to the discrete, pixelized nature of the data and the sub-pixel interpolation. As Jedrzejewski (1987) already 
pointed out, the sub-pixel interpolation scheme used can introduce spurious artefacts especially when the gradients in the luminosity profiles are large (i.e., when they are steeply cusped).

Clearly, when determining the intrinsic central cusp slopes of galaxies one can not use the surface brightness profiles determined from the isophotal analysis. We therefore use a different approach. For $r \geq 0.2^{\prime \prime}$ we fit the Nuker law profiles (equation [3]) directly to the major axis luminosity profile determined from the isophotal fit to the image. For $r<0.2^{\prime \prime}$, however, we extract the actual pixel values of the image from the data, which we compare to the model predictions given by

$$
I_{\text {fit }}(x, y)=\iint_{\text {pixel }} I(\hat{m}) \mathrm{d} \hat{x} \mathrm{~d} \hat{y},
$$

with $I(r)$ the Nuker law profile and

$$
\hat{m}^{2}=\hat{x}^{2}+\left(\frac{\hat{y}}{1-\epsilon_{0}}\right)^{2} .
$$

Here $(\hat{x}, \hat{y})$ are the coordinates centered on the galaxy center $\left(x_{0}, y_{0}\right)$ with the $x$-axis rotated by an angle $\theta_{0}$ with respect to the pixel coordinate system, and $\epsilon_{0}$ and $\theta_{0}$ are the ellipticity and position angle of the isophotes inside $0.2^{\prime \prime}$, respectively. We make the assumption that $x_{0}, y_{0}, \epsilon_{0}$, and $\theta_{0}$ are all constant inside $0.2^{\prime \prime}$ and we compute their values as the average of the corresponding isophotal parameters in the radial interval from $0.2^{\prime \prime}$ to $0.4^{\prime \prime}$. In cases where the isophotes in this radial range are not representative of the galaxy, e.g., distorted by dust, a different more appropriate radial range is chosen.

The advantage of this method is that by using the observed pixel values to determine the best fit Nuker profile we do not suffer from any problems with sub-pixel interpolation which hampers a proper isophotal analysis at small radii. The disadvantage, however, is that if the actual isophotal parameters vary with radius inside $0.2^{\prime \prime}$, this will introduce systematic errors in the parameters of the best fit Nuker law profile. However, detailed tests, described below, show that these errors are much smaller than those stemming from sub-pixel interpolation. Note that a somewhat similar scheme was used by Byun et al. (1996), who used the total integrated light inside $0.1^{\prime \prime}$ as an additional constraint for the Nuker-law fitting procedure.

Figure 3 illustrates the advantage of our new method. The solid lines correspond to the intrinsic major axis luminosity profile of the same model as in Figure 1 (i.e., with $\gamma=0.7$ ). The upper profiles with the filled circles correspond to the luminosity profiles determined from the isophotal analysis. As is evident, inside $\sim 0.1^{\prime \prime}$ the sub-pixel interpolation used by the isophotal analysis introduces systematic errors. Note again that the results for the unconvolved (left panels) and deconvolved (right panels) model images are virtually identical, indicating that the deconvolution procedure used can accurately correct for the PSF smearing. The lower profiles with open symbols, offset by 1.5 magn, correspond to the luminosity profile determined with the new method. The open circles $\left(r \geq 0.2^{\prime \prime}\right)$ are the luminosities determined from the isophotal analysis. The open squares correspond to the actual pixel values of the model image inside $0.2^{\prime \prime}$.

\footnotetext{
${ }^{5}$ Since in general the (center of the) pixels do not lie exactly along the major axis, we make use of the following
} 
As is evident from the lower panels, this new method allows us to recover the intrinsic luminosity profile with high accuracy down to very small radii.

In order to determine the parameters of the Nuker law profile that best fits the data we use a $\chi^{2}$-minimization technique. For a given set of parameters $\left(\alpha, \beta, \gamma, r_{b}, I_{b}\right)$, we determine $\chi^{2} \equiv \chi_{1}^{2}+f^{-2} \chi_{2}^{2}$. Here $\chi_{1}^{2}$ and $\chi_{2}^{2}$ are the $\chi^{2}$-values of the pixel-value fitting inside $0.2^{\prime \prime}$ and the luminosity profile fitting at $r \geq 0.2^{\prime \prime}$, respectively. As errors we use Poisson statistics for the pixel values and the nominal errors given by the isophotal analysis, respectively. In practice, these latter errors are underestimated, as is evident from the fact that the normalized $\chi_{2}^{2}$ of the best fit model is typically significantly more than unity. We therefore introduce the parameter $f$, which scales the nominal error given by the isophotal analysis, and which thus sets the relative contributions of $\chi_{1}^{2}$ and $\chi_{2}^{2}$ to the total $\chi^{2}$ of the fit. In the left panel of Figure 4 we plot the average difference between the intrinsic cusp steepness $\gamma_{\text {model }}$ and the best-fit Nuker-law profile $\gamma_{\text {fit }}$, as function of $f$ for the set of models with $\gamma=0.5$. The averages are taken over all 50 model galaxies in this set. As expected, the fits are better for $f>1$. A value of $f \simeq 5$ yields values for $\gamma_{\text {fit }}$ in good agreement with $\gamma_{\text {model }}$. For $f \lesssim 1$, too much relative weight is given to the pixel values inside $0.2^{\prime \prime}$, and $\gamma_{\text {fit }}$ is too large. For too large $f, \chi^{2}$ is completely dominated by the isophotal luminosities outside $0.2^{\prime \prime}$ and the central cusp slope becomes poorly constrained, as is evident from the large errorbars. In what follows we therefore adhere to $f=5$, for which in general we find a normalized $\chi^{2}$ of the best fit model close to unity.

We have constructed luminosity profiles for all 750 deconvolved model images using our new method, and determined the best fit Nuker law parameters using the $\chi^{2}$-minimization technique described above with $f=5$. In Figure 1 we plot $\gamma_{\text {fit }}$ versus $\gamma_{\text {model }}$ for all 750 models. As is evident, this method of analyzing luminosity profiles allows an accurate determination of the central cusp slope, and we use the same method to analyze the luminosity profiles of the actual HST images.

\section{Results}

\subsection{Isophotal Analysis}

For each galaxy there are six reduced images: the non-deconvolved and deconvolved 300 sec, $700 \mathrm{sec}$, and combined images. An isophotal analysis on each of these six images is performed. All images are checked for contamination such as cosmic rays that have not been properly removed, foreground stars, dust filaments, and bright globular clusters. If necessary, these objects are masked out. Ten galaxies are so severely influenced by dust that meaningful estimates of their luminosity profiles and isophotal structures can not be obtained (all galaxies with filamentary dust of "type" III as well as NGC 4233 and NGC 4494, which have a large dust disk, see $\S 5.3$

way to visualize the fit residuals in the luminosity profile plot: We plot the pixel values at the radius $r_{p l o t}$ defined such that $I\left(r_{p l o t}\right)=I_{f i t}(x, y)$, i.e., the difference between the pixel value and the Nuker-law profile at $r_{p l o t}$ is equal to the difference between the actual pixel value and the prediction $I_{f i t}(x, y)$ of the best-fit model. This way the residual is accurately visualized. 
below). In most of the following discussion we discard these galaxies and focus on the sample of 57 galaxies for which the HST images provide a meaningful picture of the structure of the stellar component. In what follows we refer to this sub-sample as the unperturbed sample.

Isophotal parameters are determined down to $r=0.03^{\prime \prime}$, and presented in the Appendix. Note that, although we plot the isophotal parameters down to $r=0.03^{\prime \prime}$, only the parameters for $r \gtrsim 0.2^{\prime \prime}$ are considered for further analysis. For comparison, the isophotal parameters of the deconvolved image (open circles) are overplotted with the ones of the non-deconvolved image (solid triangles). At larger radii, unaffected by convolution effects, the latter data, which is less noisy, nicely complements the deconvolved data.

Previous high-resolution imaging with the HST has revealed that the central regions of early-type galaxies are very complex environments. This is strikingly confirmed with our sample, and is clearly reflected in the isophotal parameters: the isophotal shapes can vary strongly with radius and are very different from one galaxy to the other. This makes it very hard to categorize the central regions of early-type galaxies in a small number of classes. It has become customary to classify ellipticals as either disky or boxy, depending on the sign of the $b_{4}$-parameter. This generally works well for the outer parts of ellipticals, where the changes in the isophotal shape are only modest. However, the central regions reveal a vastly more complex behavior. A single galaxy can have isophotes that change from disky to boxy and back to disky all within the inner $10^{\prime \prime}$, even when there are no signs of dust. Clearly, a bimodal classification does not suffice to describe the bewildering variety of morphologies apparent in the inner $\sim 10^{\prime \prime}$. Another approach is therefore employed: Rather than classifying galaxies as either disky or boxy a simple letter coding, referred to as isophotal code, is used to describe the individual isophotal shapes of each galaxy. The letters indicate regions with common isophotal shape, starting from the center at $r=0.2^{\prime \prime}$ out to $r \simeq 25^{\prime \prime}$, where $d, b, 0, x$ and ? refer to disky, boxy, neither disky nor boxy, undetermined due to dust, and undetermined due to small surface brightness gradient, respectively. As an example, the isophotal code $x 0 d$ for NGC 2592 indicates that for small radii the isophotal shape could not be determined due to dust (in this case a small dust disk, see $\S 5.3$ ), followed by a region where the isophotes are elliptical, and which become disky at larger radii. As a general rule, a galaxy region is classified as disky (boxy), if $b_{4}>+0.01\left(b_{4}<-0.01\right)$ for several consecutive isophotes while the other high-order parameters are not significantly different from zero. Exceptions are misaligned structures which also result in non-zero $a_{4}$ values. A more detailed description of these intriguing structures is given in $\S 5.5$.

The isophotal codes for each galaxy are listed in column (5) in Table 2. Clearly, this encoding is somewhat subjective. Nevertheless, it is well suited to describe the complex and diverse isophotal behavior of the galaxies. In addition to the isophotal code, the median ellipticity $\langle\epsilon\rangle$ calculated over the radial interval from $1.0^{\prime \prime}$ to $10.0^{\prime \prime}$ is listed in Column (8) of Table 2. Note that these median values may not be very meaningful for galaxies that have rapidly changing ellipticities. Large variations may be real or may reflect the presence of dust, and are easily identified by their relatively large errors $(\Delta\langle\epsilon\rangle \gtrsim 0.1)$. 


\subsection{Luminosity Profiles}

Luminosity profiles along both the major and minor axes of the deconvolved images are determined and subsequently fitted with the Nuker-law profile as described in detail in $\S$ 4.1. In some cases a deviation from equation (3) is visible in the inner $\sim 0.1^{\prime \prime}$ as excess light, and pixels inside $0.1^{\prime \prime}$ are ignored in the fitting procedure. These (largely unresolved) 'nuclei' are discussed in more detail in $\S 5.7$. In the cases where a central dust disk is present, we exclude any data inside the major axis radius of the dust disk from the fit. The best fit parameters are listed in Tables 3 and 4 for the major and minor axes, respectively. Plots of the luminosity profiles are presented in the Appendix, with the best fit Nuker law profiles overplotted (solid lines). In cases where the luminosity profiles do not reveal a clear change in slope over the entire radial range (NGC 4494 and NGC 3377 along the minor axis) the observed luminosity profile is fitted by a single power law:

$$
I(r)=I_{0} r^{-\beta}
$$

For these galaxies, the values listed for $I_{b}$ correspond to $I_{0}$ in Tables 3 and 4 , and no values for $\alpha$, $\gamma$ and $r_{b}$ are given.

\subsubsection{Cusps versus Cores}

As discussed in $\S$ 1, HST images have revealed that all early-type galaxies have luminosity profiles with central cusps. More importantly, it was found that the distribution of cusp slopes is bimodal and in support of the dichotomy of early-type galaxies as inferred from their isophotal and kinematical structure. In their study of the central parameter relations of early-type galaxies, Faber et al. (1997; hereafter F97) classified all galaxies with $\gamma<0.3$ and $r_{b} \geq 0.16^{\prime \prime}$ as core galaxies. All other galaxies were classified as power-law galaxies. It is important to realize that such classification scheme suffers from distance effects. Galaxies at large distances will have break radii $r_{b}$ that are smaller than the resolution limit of the simulations. Consequently, the central cusp slopes of the observed luminosity profiles of these systems are to be compared to $\beta$, and not $\gamma$, of less distant systems. Similarly, galaxies that are much closer than the main population of systems might reveal central luminosity profiles that deviate from the simple double-power law. Indeed, F97 pointed out that both M31 and M32 are poorly fit by the Nuker-law profile, and that their classification would have been different had they been at the distance of Virgo.

With these caveats in mind, we now follow F97 and attempt to classify the galaxies in our unperturbed sample as either 'core' or 'power-law'. However, when resorting to the same classification scheme as used by F97 we are confronted with another shortcoming concerning luminosity profiles having small $\alpha$. Such profiles occur in our sample for two reasons: because of the distance-problem alluded to above, or because they intrinsically have luminosity profiles for which the slope changes smoothly with radius. Some galaxies in the latter category can still be fit by a Nuker-law profile, while for others, even small $\alpha$ Nuker-law profiles fits cannot accomodate all profile features. In all of these cases we generally find best-fitting Nuker-law profiles with $\gamma \simeq 0$. However, $\gamma$ is only a proper representation of the gradient in the luminosity profile at radii $r$ for 
which $\left(r / r_{b}\right)^{\alpha} \ll 1$. For small $\alpha$, however, $\left(r / r_{b}\right)^{\alpha}$ can be close to unity, even when $r$ is significantly smaller than $r_{b}$, and $\gamma$ therefore becomes a measure of the gradient of the luminosity profile at radii much smaller than the actual resolution limit. Since one has to be sceptical about basing a galaxy's classification on extrapolated quantities, we complement our classification scheme by introducing the parameter

$$
\gamma^{\prime} \equiv-\left[\frac{\mathrm{d} \log I}{\mathrm{~d} \log r}\right]_{r=0.1^{\prime \prime}}=\frac{\gamma+\beta\left(\frac{r^{\prime}}{r_{b}}\right)^{\alpha}}{1+\left(\frac{r^{\prime}}{r_{b}}\right)^{\alpha}}
$$

with $r^{\prime}=0.1^{\prime \prime}$. Thus, $\gamma^{\prime}$ is determined directly from the best-fit Nuker-law, and is a measure of the gradient of the luminosity profile at $r=0.1^{\prime \prime}$. The values of $\gamma^{\prime}$ along the major and minor axes of the luminosity profiles are listed in Tables 3 and 4 , respectively.

In the upper panels of Figure 5 we compare the cusp slopes of the luminosity profiles along the major and minor axes. Both $\gamma$ and $\gamma^{\prime}$ give very similar results along both axes, indicating that our method of using the actual observed pixel values inside $0.2^{\prime \prime}$ to constrain the Nuker-law fit yields a robust measure of the central cusp slope. Comparing $\gamma$ to $\gamma^{\prime}$ for both the major and minor axis luminosity profiles (lower panels of Figure 5) yields a more complicated pixture. Galaxies with $\gamma>0.5$ in general have $\gamma^{\prime} \simeq \gamma$. However, for $\gamma<0.3$ about half of the galaxies have $\gamma^{\prime}<0.3$, and the other half has $\gamma^{\prime}>0.3$. We therefore use the following classification scheme, based on both $\gamma_{\text {maj }}$ and $\gamma_{\text {maj }}^{\prime}$ ?: core galaxies have $\gamma_{\text {maj }}^{\prime}<0.3$, power-law galaxies have $\gamma_{\text {maj }} \geq 0.3$, and galaxies with $\gamma_{\text {maj }}^{\prime} \geq 0.3$ and $\gamma_{\text {maj }}<0.3$ are provisionally refered to as 'intermediate' galaxies. Note that this classification scheme is more conservative in designating galaxies as core galaxies as the scheme used by F97: In our sample, only 56 percent of the galaxies classified as core galaxies according to the F97 scheme are classified similarly with our definition. The other 44 percent are classified as intermediate. Column (2) in Table 2 indicates the classification of our galaxies as either core ' $\cap$ ', power-law ' $\backslash$ ', or intermediate ')'. A question mark indicates that the galaxy is not in the unperturbed sample, and that no attempt has been made to classify this galaxy.

Figure 6 presents histograms of the distributions of $\gamma$ and $\gamma^{\prime}$. Although the distribution of cusp slopes reveals a clear hint for bimodality, consistent with the results of F97, we find less of a deficit of galaxies with $0.3 \leq \gamma \lesssim 0.5$. Most of the galaxies with $\gamma$ in this range, and which are thus classified as power-law galaxies (e.g., NGC 5576, NGC 5796, and NGC 5831), have small values of $\alpha$ and no disky isophotes, similar to the intermediate galaxies. The intermediate galaxies themselves follow the same distribution of $\gamma$ as the core galaxies, but at the same time occupy the low- $\gamma^{\prime}$ end of the power-law galaxies. Thus, whereas we confirm the bimodality in cusp slopes found by F97, we find that $\sim 15$ percent of the galaxies in our unperturbed sample have luminosity profiles whose profile slopes change very gradually with radius (reflected by the small value of $\alpha$ ), making a classification based on central cusp slope somewhat ambiguous (see discussion in $\S 5.4 .2$ below).

\footnotetext{
${ }^{6}$ our results would be identical had we used $\gamma_{\min }$ and $\gamma_{\min }^{\prime}$
} 


\subsection{Dust Properties}

As discussed in the introduction, the high resolution images obtained with the HST have strongly increased the detection rate of dust in early-type galaxies. Two different dust morphologies are distinguished: disks (or tori) and filaments (indicated by 'd' and 'f', respectively, in Table 21). The amount of filamentary dust present is indicated by a Roman numeral, ranging from I (small traces of dust that hardly influence the isophotal shapes) to III (large amounts of dust that prevent a meaningful analysis of the isophotes and luminosity profiles). Types II and III are easily discerned from an inspection of the images by eye, whereas filamentary dust of class I is only detected after a more detailed investigation. A detailed discussion of the dust properties in our sample is presented in Tran et al. (2000). Here we merely list the main statistics.

In 10 galaxies (15 percent) we find a nuclear dust disk, with a diameter ranging from $0.3^{\prime \prime}$ (NGC 4648) to $12.3^{\prime \prime}$ (NGC 4233). Most remarkably, 8 of these galaxies are classified as power-law galaxies, whereas the two remaining, NGC 4233 and NGC 4494, have a dust disk that is too large to be able to classify the galaxy based on its luminosity profile. Filamentary dust is found in 19 cases (28 percent). In total, we thus find evidence for dust in 43 percent of our galaxies. Van Dokkum \& Franx (1995), analyzing 64 early-type galaxies imaged with the HST before the refurbishment mission, found dust in 48 percent of the cases and pointed out that a significant fraction of the dust will be missed because of inclination effects. They estimated that $\sim 75$ percent of the early type galaxies harbor significant amounts of dust. Our results are in good agreement with their estimates.

\subsection{The dichotomy amongst early-type galaxies}

\subsubsection{Isophotal shapes}

As mentioned in the introduction, early-type galaxies come in two different classes, core and power-law galaxies. The latter are relatively faint $\left(M_{B} \gtrsim-20.5\right)$, typically have disky isophotes, and are thought to be rotationally supported spheroidal systems. Core galaxies, on the other hand, are relatively bright systems, often with boxy isophotes, that are thought to be triaxial and henceforth pressure-supported. We now investigate to what extent the galaxies in our sample obey this dichotomy. As discussed in $\S$ 5.1, the isophotal structures of the central regions are very complex, and we introduced an isophotal code (hereafter IC) to describe the observed morphologies. The galaxies can roughly be divided into four classes: disky ('d', ICs with $d$ but no $b$ ), boxy ('b', ICs with $b$ but no $d$ ), disky-boxy ('db', ICs with both $d$ and $b$ ), and regular ('0', ICs with no $d$ or $b$ ).

In Table 5 we correlate this classification based on the isophotal structure with that based on the luminosity profiles. We only consider the 57 galaxies in our unperturbed sample. In general, our sample nicely confirms the dichotomy that has emerged from previous studies (e.g., Jaffe et al. 1994; Lauer et al. 1995): 20 of the 21 disky ('d') galaxies are classified as power-laws, while 7 of the 9 core galaxies are classified as either boxy ('b') or regular (' 0 '). However, not all 
galaxies fall in either of these two classes. As we indicated in $\S$ 5.2.1, roughly twelve percent of our galaxies classify as 'intermediate' based on their luminosity profiles. Remarkably, none of these galaxies reveal any sign of diskiness. In fact, two of them classify as boxy, whereas the other four are regular. As such, these intermediate galaxies are more similar to core galaxies than to power-law galaxies. Of the in total 8 galaxies with both disky and boxy isophotes ('db'), 7 classify as power-law galaxies. In total, we thus find that 93 percent of all galaxies with disky isophotes are power-law galaxies. If one takes into account that nuclear disks are much easier detectable in core galaxies than in power-law galaxies, it is clear that there is a very strong correlation between the diskiness and central cusp slope of early-type galaxies.

Four of the in total 57 galaxies in our unperturbed sample deviate from these general trends: NGC 3078 and NGC 5576 are the only power-law galaxies (out of 41) with boxy isophotes. NGC 3078 is also the galaxy with the highest $1.4 \mathrm{GHz}$ flux in our sample, and reveals a central dust disk with a diameter of $\sim 1.4^{\prime \prime}$. The dust absorption combined with the presence of a strong AGN makes the classification rather uncertain, and it is thus not inconceivable that NGC 3078 is in fact a core galaxy. NGC 5576, on the other hand, has no detectable dust or strong radio emission. However, with $\gamma_{\mathrm{maj}}=0.36$ and $\alpha=0.79$ it is close to an intermediate galaxy. NGC 3613 and NGC 4365 are the only two core galaxies with disky isophotes: NGC 3613 harbours a thin edge-on nuclear disk inside the central arcsec, and NGC 4365 contains a kinematically decoupled core with the characteristics of a cold, rotating system (Surma 1993).

\subsubsection{Central cusp slopes}

In Figure 7 we plot $\gamma_{\text {maj }}^{\prime}$ as function of both the (major axis) break radius $r_{b}$ and the absolute $B$-band magnitude $M_{B}$. Solid circles correspond to core galaxies, solid squares to power-law galaxies, and open circles to intermediate galaxies. All 10 core galaxies have break radii in the (relatively narrow) interval $0.3^{\prime \prime} \lesssim r_{b} \lesssim 2^{\prime \prime}$, and are bright $\left(M_{B} \lesssim-20\right)$. The power-law galaxies, on the other hand, typically have $M_{B} \gtrsim-20$ and $\gamma_{\text {maj }}^{\prime} \gtrsim 0.6$. This once again confirms the dichotomy amongst early-type galaxies inferred from previous studies.

But what about the intermediate galaxies? According to their isophotal structure, one would tend to classify them as core galaxies (see $\S$ 5.4.1). The same is true if one bases the classification on $\gamma$ (cf. Figure 6). However, they also have properties that clearly differentiate them from core galaxies: their $\gamma^{\prime}$-values are more similar to those of the power-law galaxies (although they occupy the low- $\gamma_{\text {maj }}^{\prime}$ end of the distribution), their luminosity profiles have values of $\alpha$ that are significantly lower than the typical value for core galaxies, and finally, their average absolute magnitudes seem to be intermediate between those of both the core and power-law galaxies?: they can not be unambiguously classified as either one of those. More detailed investigations of their properties are required to see if they make up a truly, physically distinct class of objects, or whether these galaxies are distinct from both the core and power-law galaxies only because of distance and/or resolution effects.

\footnotetext{
${ }^{7}$ except for MCG 11-14-25A, which is the faintest galaxy in our sample with $M_{B}=-17.90$
} 


\subsection{Misaligned Structures}

In fourteen galaxies we find radial intervals with strong isophote twists, non-zero fourth order moments $\left(a_{4}\right.$ and/or $\left.b_{4}\right)$ and local ellipticity maxima. A clear example is NGC 2699 where at $\sim 4^{\prime \prime}$ from the center the isophotes reveal an isophote twist of $\sim 25^{\circ}$, both $a_{4}$ and $b_{4}$ are positive, and there is a local maximum in ellipticity. In some cases the orientation angle of the misaligned structures changes with radius, giving it the appearance of spiral arms (e.g., ESO 443-39, NGC 3595, and UGC 4551), wheras in other cases (ESO 378-20, ESO 443-39, ESO 447-30, NGC 2950 and UGC 6062) misaligned structures are evident in two distinct radial intervals. A clear example is ESO 447-30 with misaligned structures at both $\sim 2.5^{\prime \prime}$ and $\sim 10^{\prime \prime}$. In each of these galaxies, the two misaligned structures are not only misaligned with the host galaxy, but also with each other.

Given the highly elongated nature of these structures and the fact that disks are common in elliptical galaxies, it is tempting to interpret these structures as stellar disks whose orientation axes are misaligned with those of the host galaxy. However, disks in spheroidal systems can only be stable if their symmetry axis is perpendicular to the equatorial plane of the potential, which does not result in a projected misalignment as observed. If, on the other hand, a stellar disk is embedded in a triaxial galaxy, projection effects can easily accommodate the observed misaligned morphologies. Furthermore, if the axis ratios of the triaxial host galaxy vary with radius, the projected angle of misalignment can vary with projected radius. Such configuration, therefore, can explain the multiple misaligned structures observed. A problem with this explanation, however, is that all 14 galaxies with misaligned structures are power-law galaxies which are generally thought

to be spheroidal systems. Furthermore, if these structures are disks the observed fraction of disks embedded in power-law galaxies is too high (see $\S 5.6$ below).

A more likely explanation, therefore, is that these misaligned structures correspond to bars. This lends great support from the fact that isophotal structures, very similar to those seen here, have been observed in barred spirals and S0s (e.g., Wozniak et al. 1995; Friedli et al. 1996; Erwin \& Sparke 1999). In particular, the bars within bars found by these authors provide a natural explanation for the multiple misaligned structures found in some of our galaxies. Indeed, both Kormendy (1981) and Wozniak et al. (1995) have previously noted the complex morphological structure of NGC 2950 (one of the fourteen galaxies in our sample with misaligned structures) and classified it as a double-barred galaxy. The bar interpretation is further supported by the fact that ten of the fourteen galaxies in our sample with misaligned structures are classified as S0 or E/S0 (see Table 1). It is unclear, however, how to interpret the misaligned structures in the four galaxies classified as ellipticals, although it is not inconceivable that these galaxies may be misclassified S0s seen close to face-on. Detailed investigations into the kinematics of these galaxies are required to confirm that indeed these structures correspond to tumbling triaxial potentials. 


\subsection{Stellar Disks}

In total 29 out of the 57 galaxies (51 percent) in our unperturbed sample reveal disky isophotes over at least a small radial interval (this includes the misaligned structures discussed in $\S$ 5.5). This diskiness is most straightforwardly interpreted as due to an embedded stellar disk (e.g., Scorza \& Bender 1995, and references therein).

The diversity of disk structures in our sample is large, ranging from extremely thin and small disks in the very center (i.e., NGC 3613) up to very large disks spanning the entire radial interval imaged (i.e., NGC 4621). In 18 galaxies the isophotes are disky in only a single radial interval, whereas the other 11 galaxies have disky isophotes in two radial intervals, separated by purely elliptical or boxy isophotes. This "double-diskiness" may be due to a nuclear disk inside the central hole of a separate outer disk or ring (i.e., van den Bosch \& Emsellem 1998), reflect the projected surface brightness contribution of a single disk which is locally dwarfed by that of the spheroidal, or be due to a bar-within-a-bar structure (see $\S$ 5.5). The observed isophotal structure of an elliptical galaxy with an embedded stellar disk depends strongly on the ratio of the surface brightness distributions of the two components and on the inclination angle of the disk. Detailed disk-bulge decompositions (i.e., Scorza \& Bender 1995; Scorza \& van den Bosch 1998) are required to decompose the observed surface brightness in the separate components, which is beyond the scope of this paper.

Given the large fraction of early-type galaxies that reveal evidence for an embedded disk, and the fact that these disks are only visible if the disk is seen under a sufficiently large inclination angle, implies that the true fraction of early-type galaxies with embedded stellar disks is likely to be significantly larger than the 51 percent found here. In our unperturbed sample, 66 percent of all power-law galaxies have some amount of diskiness. This is a surprisingly high fraction. If we assume that all power-law galaxies have embedded disks, and that these disks are only detectable if their projected minor-to-major axis ratio is smaller than 0.5 , one only expects to detect disks in 33 percent of all power-law galaxies (see Rix \& White 1990 for a detailed discussion on the detectability of embedded stellar disks). The observed fraction of 66 percent implies either of the following: (i) all disks with a projected axis ratio smaller than 0.86 are detectable (assuming that all power-law galaxies harbor embedded disks), (ii) power-law galaxies are always seen under high inclination angles, (iii) the steep central cusp owes to the projection of the highly flattened structure (but see Lauer et al. 1995; Faber et al. 1997), or (iv) in some cases the diskiness is due to highly elongated prolate or triaxial structures (i.e., bars) rather than oblate disks.

We only consider the latter of these explanations viable. It is supported by the fact that fourteen of our power law galaxies have their diskiness misaligned with the main galaxy. As we discussed in the previous section, these structures are most likely due to bars in S0 galaxies. If we exclude these fourteen galaxies, we find embedded disks in only 32 percent of our power-law galaxies, which is in excellent agreement with the 33 percent expected if all power-law galaxies harbor embedded disks, but which are only detectable if the disk's projected axis ratio is smaller than 0.5 . 


\subsection{Nucleated Galaxies}

Although the Nuker-law in general provides a good fit to the observed surface brightness of the central regions of early-type galaxies, in several cases the very centers reveal excess light, associated with an increase in slope as the HST resolution limit is approached. We follow Lauer et al. (1995) and refer to these features as nuclei. These unresolved nuclei may be due to non-thermal emission from AGNs or may reflect the presence of a nuclear star cluster.

Table 2 lists the degree of nucleation observed, ranging from very mild, indicated by 'I' (e.g., NGC 2634) to nuclei that stand out strongly against the 'background' Nuker-law profile, indicated by 'III' (e.g., NGC 4482). In order to be termed 'nucleated' we require that the central steepening of the surface brightness occurs inside $0.15^{\prime \prime}$, and is visible from both the major and minor axes surface brightness profiles. Note that effects due to deconvolution as well as dust obscuration can produce artefacts in the luminosity profiles that are similar to our level I nucleation. In order to guard against such artificial 'detections', we have been fairly conservative in assigning nucleation.

In total, nuclei are detected in 9 out of the 67 galaxies (13 percent) in our sample. This is less than half the detection rate of Lauer et al. (1995), who detected nuclei in 16 out of 45 galaxies. Except for the fact that we have been fairly conservative in assigning nuclei, it is unclear why our detection rate is so much smaller. Only 2 of the 16 nucleated galaxies in the sample of Lauer et al. are core galaxies. In both cases the nucleation is interpreted as non-thermal emission from AGNs. In our sample, the two nucleated galaxies (NGC 4168 and NGC 5077) not classified as power-law galaxies have been detected at $1.4 \mathrm{GHz}$ (see Table 1). This is consistent with the idea that 'nucleation' in core (and intermediate) galaxies is associated with an active galactic nucleus. For none of the 7 nucleated power-law galaxies do we find any sign of non-thermal emission associated with an AGN (i.e., none of these galaxies have been detected at $1.4 \mathrm{GHz}$ ). This again is in good agreement with the results of Lauer et al. (1995). These results, however, are somewhat puzzling from an astrophysical point of view. An often advertised interpretation of (non-AGN related) nuclei is that they are stellar remnants of cannibalized, low-mass galaxies that have reached the center by means of dynamical friction. Since less luminous galaxies are in general denser than their more massive counterparts, one expects to find stellar nuclei predominantly in the less dense cores of the more massive galaxies, where tidal forces are less severe (e.g., Kormendy 1984; Faber et al. 1997). The almost complete absence of stellar nuclei in core galaxies hints at some violent disruption process, most likely associated with massive black holes (see Merritt \& Cruz 2001, and references therein). However, this fails to explain why power-law galaxies do seem to harbor stellar nuclei. These galaxies have massive black holes as well, and their intrinsically high stellar densities will only aid in disrupting any merger remnant that might reach the center of the potential well. A more viable explanation is that these nuclei are the product of some dissipational formation mechanism. The presence of highly flattened disk-structures in the majority of these power-law galaxies supports the view that dissipational processes have played an important role. 


\section{Conclusions}

We have reported on the $R$-band images of the central regions of 67 early-type galaxies obtained with the WFPC2 aboard the HST. This sample roughly doubles the number of early-type galaxies that have now been imaged at HST resolution, and complements similar data on the central regions of radio galaxies and the bulges of spiral galaxies. In this paper we have presented the data, focusing in particular on the isophotal structures and luminosity profiles.

Our sample strikingly confirms the complex morphologies of the stellar component in the central regions of early-type galaxies which have become apparent from previous studies with HST. Whereas the outer regions of early-type galaxies can in general be classified as either disky or boxy, the isophotal structure in the inner $\sim 10^{\prime \prime}$ varies strongly with radius, such that a bimodal classification does not suffice to describe the bewildering variety of structures seen. Instead, we have used a simple letter coding to describe the diverse morphologies.

Previous imaging surveys with HST have shown that a more suitable criterion for classifying the central regions of early-type galaxies is based on their luminosity profiles (rather than their isophotal structure). Depending on the slope of the central cusp, galaxies are classified as either a core galaxy or a power-law galaxy. We determined the luminosity profiles along the major and minor axes for 57 galaxies in our sample (the unperturbed sample). The remaining 10 galaxies do not allow a proper assessment of their luminosity profiles because of dust obscuration. We have shown how luminosity profiles derived from an isophotal analysis of the data can produce relatively large systematic errors in the central region, owing to the sub-pixel interpolation used. We therefore use the actual pixel values in the central $0.2^{\prime \prime}$ to define the luminosity profiles. We fit the luminosity profiles with a Nuker-law profile (equation [3]), which has been shown to adequately describe the luminosity profiles of early-type galaxies in the inner $\sim 20^{\prime \prime}$. At radii less than $0.2^{\prime \prime}$ we fit this Nuker-law model directly to the observed pixel values, while at larger radii we use the luminosity profile as determined from the isophotal analysis. Detailed modeling has shown that this method allows an accurate recovery of the actual luminosity profiles.

In a similar study, based on a sample of 61 early-type galaxies imaged with the WFPC1, Faber et al. (1997) classified galaxies as core galaxies if the central cusp slope $\gamma$ of the best fitting Nuker law was less than 0.3. Galaxies with $\gamma>0.3$ were classified as power-law galaxies. In our sample we find a subset of galaxies with luminosity profiles that have a continuously changing slope, rather than a double power-law shape with a clear break radius. For these systems $\gamma$ of the best-fit Nuker-law profile is smaller than 0.3 , but it is only a measure of the gradient of the luminosity profile at radii much smaller than the resolution limit of the actual observations. At the resolution limit the actual slope of the luminosity profile is steeper than 0.3 , and it is unclear whether to classify these systems as core or power-law galaxies. Although the isophotal properties are similar to those of the core galaxies, there is a hint that the luminosities of these galaxies are intermediate between those of the core and power-law galaxies. We therefore provisionally classify these galaxies as 'intermediate'. However, it is unclear at present whether these galaxies make up a physically distinct class or whether distance and/or resolution effects cause them to loose their distinct core and/or power-law characteristics. 
F97 have pointed out that the distribution of central cusp slopes is clearly bimodal: whereas (by definition) core galaxies have $\gamma<0.3$, power-law galaxies are found to have $\gamma \gtrsim 0.5$. The region $0.3<\gamma<0.5$ is virtually devoid of galaxies. This bimodality closely follows the dichotomy in other properties: the core galaxies make up the bright end of the luminosity function and typically have boxy isophotes, whereas the power-law galaxies are fainter and typically have disky isophotes. We confirm this dichotomy with our sample: 93 percent of all galaxies that reveal disky isophotes are power-law galaxies, whereas 77 percent of all core galaxies have either boxy or purely elliptical isophotes. Furthermore, we confirm the bimodality of cusp slopes, but we do find a significant population of galaxies with $0.3<\gamma<0.5$. Combined with the class of intermediate galaxies, which have cusp slopes at the resolution limit that also fall in this range, we thus conclude that the bimodality is not as strong as previously claimed.

Finally, the statistical properties of our sample can be summarized as follows:

- In 15 percent of the galaxies in our sample we detect dust disks with diameters that range from $0.3^{\prime \prime}$ to $12.3^{\prime \prime}$. Together with the 28 percent of the galaxies in which we detect filamentary dust, the total detection rate of dust is 43 percent, in good agreement with previous estimates based on HST data (van Dokkum \& Franx 1995).

- In 9 galaxies we find evidence for nuclei. These are defined as (largely) unresolved, nuclear surface brightness spikes that cause a central upturn in the luminosity profiles. Our detection rate of nuclei (13 percent) is substantially less than the 36 percent found by Lauer et al. (1995) in their sample, but this is most likely a reflection of our more conservative approach to identifying nucleation. Whereas the nuclei in power-law galaxies are most likely stellar, the nucleation seen in core galaxies is generally non-thermal emission from an active galactic nucleus.

- In 14 galaxies we find structures that are misaligned with the principal axes of the host galaxy. These structures are most likely associated with stellar bars in S0 galaxies. In 5 of these galaxies, multiple misaligned structures are apparent indicating bars within bars.

- A large fraction (51 percent) of the early-type galaxies in our unperturbed sample reveal disky isophotes over at least a small radial interval, whereas this fraction increases to almost 70 percent when only considering power-law galaxies. This is a remarkably large fraction: even if all power-law galaxies were to harbor an embedded stellar disk, one would not expect to detect these thin structures in more than $\sim 30$ percent of all cases because of inclination effects. If, however, we exclude the cases where the diskiness is misaligned with the main galaxy, and which are more likely associated with stellar bars, we find that $\sim 32$ percent of all power-law galaxies reveal some amount of diskiness. This is consistent with a picture in which all power-law galaxies harbor an embedded stellar disk.

We are grateful to the referee, Tod R. Lauer, for his insightful comments that greatly improved the analysis and presentation of the data in this paper. The observations presented in this paper were obtained with the NASA/ESA Hubble Space Telescope, which is operated by 
AURA, Inc., under NASA contract NAS 5-26555. We acknowledge the use of the LEDA database (www-obs.univ-lyon1.fr). FvdB was supported by NASA through Hubble Fellowship grant \# HF-01102.11-97.A awarded by the Space Telescope Science Institute. 


\section{A. Contour maps, luminosity profiles, and isophotal parameters}

In the following figures we present the data for all 67 galaxies in our sample. The upper right-hand panels show contour plots of the $33.0^{\prime \prime} \times 33.0^{\prime \prime}$ field of view of the Planetary Camera. The thick black bar in the upper-right corner corresponds to an angular size of $5.0^{\prime \prime}$. Contours are plotted at intervals of 0.3 mag between $\mu_{R}=22.5 \mathrm{mag} \operatorname{arcsec}^{-2}$ and the surface brightness at $r=1.0^{\prime \prime}$ (in order to prevent central crowding of contours).

The upper-panels on the left show the observed luminosity profiles (open circles are determined from the isophotal analysis, closed circles are the actual pixel values fitted and plotted as described in $\S$ 4.1) along the major (upper curves) and minor axis (lower curves, offset by $+0.5 \mathrm{mag}$ for clarity). The thick solid lines are the best fit Nuker-law profiles, the parameters of which are listed in Tables 3 and 4 . In some cases data in the central regions is excluded from the Nuker-law profile fit because of nucleation and/or nuclear dust disks. In these cases a vertical thin dashed line indicates the lower limit of the data range used.

The remaining panels plot the isophotal parameters for both the non-deconvolved image (solid triangles) and the deconvolved image (open circles with errorbars). For each galaxy we plot as function of radius the ellipticities $(\epsilon)$, the position angles of the isophotes $(\theta$, measured from North to East), and the higher-order parameters $a_{n}$ and $b_{n}$ (with $n=3,4$ ).

Some galaxies have their central regions saturated in the $700 \mathrm{sec}$ exposure. In these cases we plot the isophotal parameters of the $300 \mathrm{sec}$ exposures in the radial regime where the 700 sec exposure shows saturation effects (rather than those of the combined image). In three cases (NGC 2950, NGC 3377, and NGC 4621), the central regions of the 300 sec exposures are also saturated. For these galaxies, only the isophotal parameters are plotted down to the radius where saturation effects in the $300 \mathrm{sec}$ exposure start. 


\section{REFERENCES}

Begelman, M. C., Blandford, R. D., \& Rees, M. J. 1980, Nature, 287, 307

Bender, R. 1988, A\&A, 193, L7

Bender, R., Surma, P., Döbereiner, S., Möllenhoff, C., \& Madejesky, R. 1989, A\&A, 217, 35

Biretta, J. A., et al. 1996, WFPC2 Instrument Handbook, Version 4.0 (Baltimore: STScI)

Bower, G. A., et al. 1998, ApJ, 492, L111

Byun, Y.-I., et al. 1996, AJ, 111, 1889

Carollo, C. M., Franx M., Illingworth, G. D., \& Forbes, D. A. 1997a, ApJ, 481, 710

Carollo, C. M., Stiavelli, M., de Zeeuw, P. T., \& Mack, J. 1997b, AJ, 114, 2366

Carollo, C. M., Stiavelli, M., \& Mack, J. 1998, AJ, 116, 68

Condon, J. J., Cotton, W. D., Greisen, E. W., Yin, Q. F., Perley, R. A., Taylor, G. B., \& Broderick, J. J. 1998, AJ, 115, 1693.

Crane, P., et al., 1993, AJ, 106, 1371

Cretton, N., \& van den Bosch, F. C. 1999, ApJ, 514, 704

Davies, R. L., Efstathiou, G., Fall, S. M., Illingworth, G., \& Schechter, P. L. 1983, ApJ, 266, 41

de Koff, S. et al. 1996, ApJS, 107, 621

de Vaucouleurs, G., de Vaucouleurs, A., \& Corwin, H. G. 1976, Second Reference Catalogue of Bright Galaxies, (Austin: University of Texas Press)

de Vaucouleurs, G., de Vaucouleurs, A., Corwin, H. G., Buta, R. J., Paturel, G., \& Fouqué, P. 1991, Third Reference Catalogue of Bright Galaxies, (Springer-Verlag)

Erwin, P., \& Sparke, L. S. 1999, ApJ, 521, L37

Faber, S. M., et al. 1997, AJ, 114, 1771 (F97)

Ferrarese, L., van den Bosch, F. C., Jaffe, W., Ford, H. C., \& O’Connell, R. W. 1994, AJ, 108, 1598

Ferrarese, L., Ford, H. C., \& Jaffe, W. 1996, ApJ, 470, 444

Friedli, D., Wozniak, H., Rieke, M., Martinet, L., \& Bratschi, P. 1996, A\&AS, 118, 461

Ford, H. C., Tsvetanov, Z. I., Ferrarese, L., \& Jaffe, W. 1998, IAU Symp. 184: The Central Regions of the Galaxy and Galaxies, ed. Yoshiaki Sofue (Dordrecht: Kluwer), 184, p. 377

Gebhardt, K., et al. 1996, AJ, 112, 105

Harms, R. J. et al. 1994, ApJ, 435, L35

Ho, L. 1999, ASSL Vol. 234: Observational Evidence for the Black Holes in the Universe, ed. S. K. Chakrabarti (Dordrecht: Kluwer), p. 157

Holtzman, J., et al. 1995a, PASP, 107, 156

Holtzman, J., et al. 1995b, PASP, 107, 1065 
Jaffe, W., Ford, H. C., Ferrarese, L., van den Bosch, F. C., \& O'Connell, R. W. 1994, AJ, 108, 1567

Jedrzejewski, R. I. 1987, MNRAS, 226, 747

Knapp, G. R., Guhathakurta, P., Kim, D.-W., \& Jura, M. A. 1989, ApJS, 70, 329

Kormendy, J. 1981, in The Structure and Evolution of Normal Galaxies, eds. M. Fall \& D. Lynden-Bell, (Cambridge Univ. Press: Cambridge), p. 85

Kormendy, J. 1984, ApJ, 287, 577

Kormendy, J. 1985, ApJ, 292, 9

Kormendy, J., Dressler, A., Byun, Y. I., Faber, S. M., Grillmair, C., Lauer, T. R., Richstone, D., \& Tremaine, S., 1994, in Dwarf Galaxies, eds. G. Meylan \& P. Prugniel (ESO Conference and Workshop Proceedings), p.147

Kormendy, J., \& Richstone, D. 1995, ARA\&A, 33, 581

Krist, J. E. 1992, The Tiny Tim User's Manual, Version 1.0, (Baltimore: STScI)

Lauer, T. R. 1985, ApJ, 292, 104

Lauer, T. R., et al. 1991, ApJ, 369, 41

Lauer, T. R., et al. 1992, AJ, 103, 703

Lauer, T. R., et al. 1995, AJ, 110, 2622

Lauer, T. R., Faber, S. M., Ajhar, E. A., Grillmair, C. J., \& Scowen, P. A., 1998, AJ, 116, 2263

Lucy, L. B. 1974, AJ, 74, 745

Macchetto, F., Marconi, A., Axon, D. J., Capetti, A., Sparks, W. B., \& Crane, P. 1997, ApJ, 489, 579

Macchetto, F. D. 1999, Ap\&SS, 269, 269

Makino, J., \& Ebisuzaki, T. 1996, ApJ, 465, 527

Martel, A. R., et al. 1999, ApJS, 122, 81

McCarthy, P. J., et al. 1997, ApJS, 112, 415

Merritt, D., \& Cruz, F. 2001, preprint astro-ph/0101194

Nieto, J.-L., Capaccioli, M., \& Held, V. E., 1988, A\&A, 195, L1

Paturel, G. et al. 1997, A\&AS, 124, 109

Pellegrini, S. 1999, A\&A, 351, 487

Phillips, A. C., Illingworth, G. D., MacKenty, J. W., \& Franx, M. 1996, AJ, 111, 1566

Quillen, A. C., Bower, G. A., Stritzinger, M. 2000, ApJS, 128, 85

Quinlan, G. D. 1996, New Astronomy, 1, 35

Quinlan, G. D., \& Hernquist, L. 1997, New Astronomy, 2, 533

Rix, H.-W., \& White, S. D. M. 1990, ApJ, 362, 52 
Scorza, C., \& Bender, R. 1995, A\&A,293, 20

Scorza, C., \& van den Bosch, F. C. 1998, MNRAS, 300, 469

Surma, P. 1993, in Structure, Dynamics, and Chemical Evolution of Elliptical Galaxies, ESO conference and Workshop Proceedings No. 45, eds. I. J. Danziger, W.W. Zeilinger, \& K. Kjär, p. 669

van den Bosch, F. C., Ferrarese, L., Jaffe, W., Ford, H. C., \& O’Connell, R. W. 1994, AJ, 108, 1579

van den Bosch, F. C., \& de Zeeuw, P. T. 1996, MNRAS,283, 381

van den Bosch, F. C., \& Emsellem, E. 1998, MNRAS,298, 267

van den Bosch, F. C., Jaffe, W., \& van der Marel, R. P. 1998, MNRAS, 293, 343

van der Marel, R. P. 1999a, AJ, 117, 744

van der Marel, R. P. 1999b, IAU Symp. 186: Galaxy Interactions at Low and High Redshift, eds J. E. Barnes, D. B. Sanders (Kluwer: Dordrecht), 186, p. 333

van der Marel, R. P., \& van den Bosch, F. C. 1999, AJ, 116, 2220

van Dokkum, P. G., \& Franx, M. 1995, AJ, 110, 2027

Verdoes Kleijn, G. A., Baum, S. A., de Zeeuw, P. T., \& O’Dea, C. P. O. 1999, AJ, 118, 2592

Wozniak, H., Friedli, D., Martinet, L., Martin, P., \& Bratschi, P. 1995, A\&A, 111, 115 


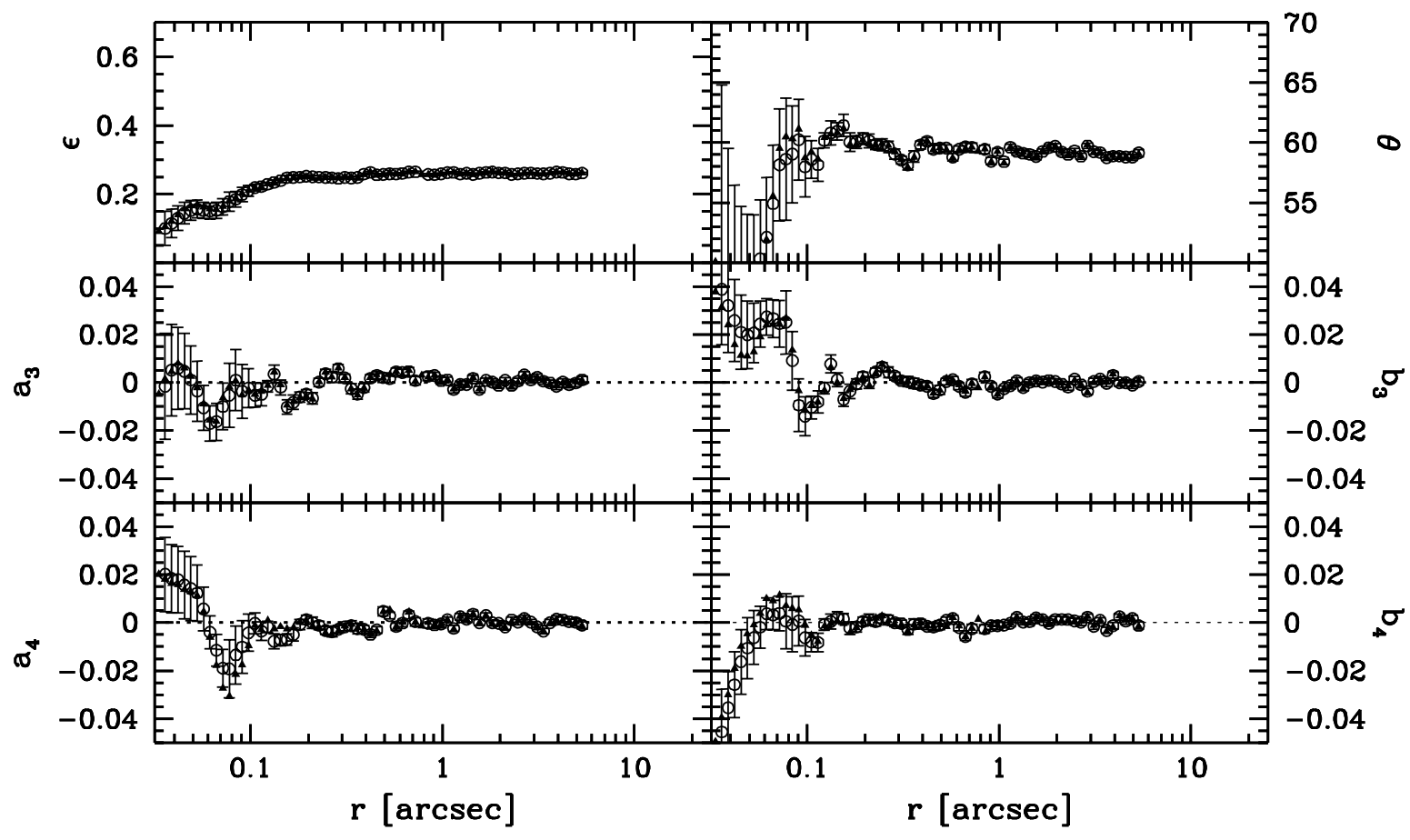

Fig. 1.- Isophotal parameters as a function of radius for a model galaxy which has perfectly elliptical isophotes with $\epsilon=0.26$ independent of radius, and a central cusp with $I(r) \propto r^{-0.7}$ (cf. Figure 3i). Solid triangles correspond to the parameters of the (unconvolved) raw image, in which only Poisson noise is introduced. The open circles with error bars correspond to the parameters of the deconvolved image. Note that for $r \gtrsim 0.2^{\prime \prime}$ the parameters of the raw and deconvolved images are in excellent agreement. We therefore are confident that we can trust the isophotal parameters of our deconvolved images at least for semi-major axis radii larger than $\sim 0.2^{\prime \prime}$. For $r \lesssim 0.2^{\prime \prime}$, however, the sub-pixel interpolation of the fitting-routine systematically underestimates the ellipticity of the isophotes, and causes artificial deviatations from their perfectly elliptical shapes. 


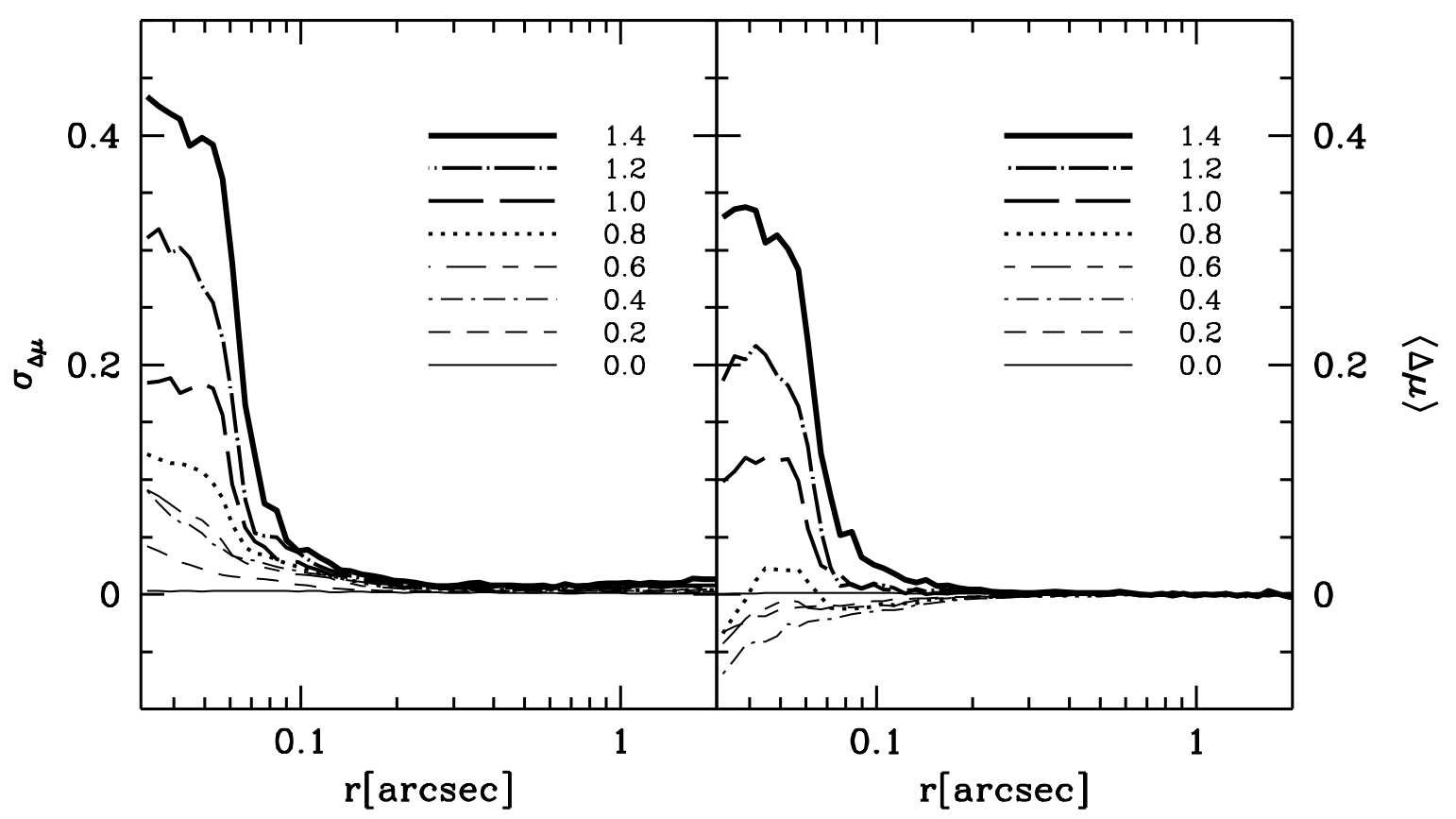

Fig. 2. - Characterization of errors in luminosity profiles. For each model $i$, the difference $\Delta \mu_{i}(r)$ between the input surface brightness profile (i.e., equation [⿶]) and the major axis luminosity profile determined from the isophotal analysis of the unconvolved image is calculated. For a sample of 8 sets of model galaxies (each set is characterized by the same value of $\gamma$ ) the average $\langle\Delta \mu\rangle(r)$ (right panel) and the corresponding standard deviation $\sigma_{\Delta \mu}(r)$ (left panel) is then computed, where the average is over the 50 models per set. The number in the legend corresponds to the characteristic central cusp slope $\gamma$ of the specific set. For $r \gtrsim 0.2^{\prime \prime}$ the luminosity profiles derived from the isophotal analysis are in excellent agreement with the intrinsic profile. For smaller radii, however, the error increases strongly, especially for the profiles with steep central cusp slopes. Note also that the average residual $\langle\Delta \mu\rangle(r)$ strongly deviates from zero at small radii, implying that the main contribution to the error is of systematic rather than random nature. This is a reflection of the problems with the isophotal analysis due to the discrete, pixelized nature of the data and the sub-pixel interpolation. 


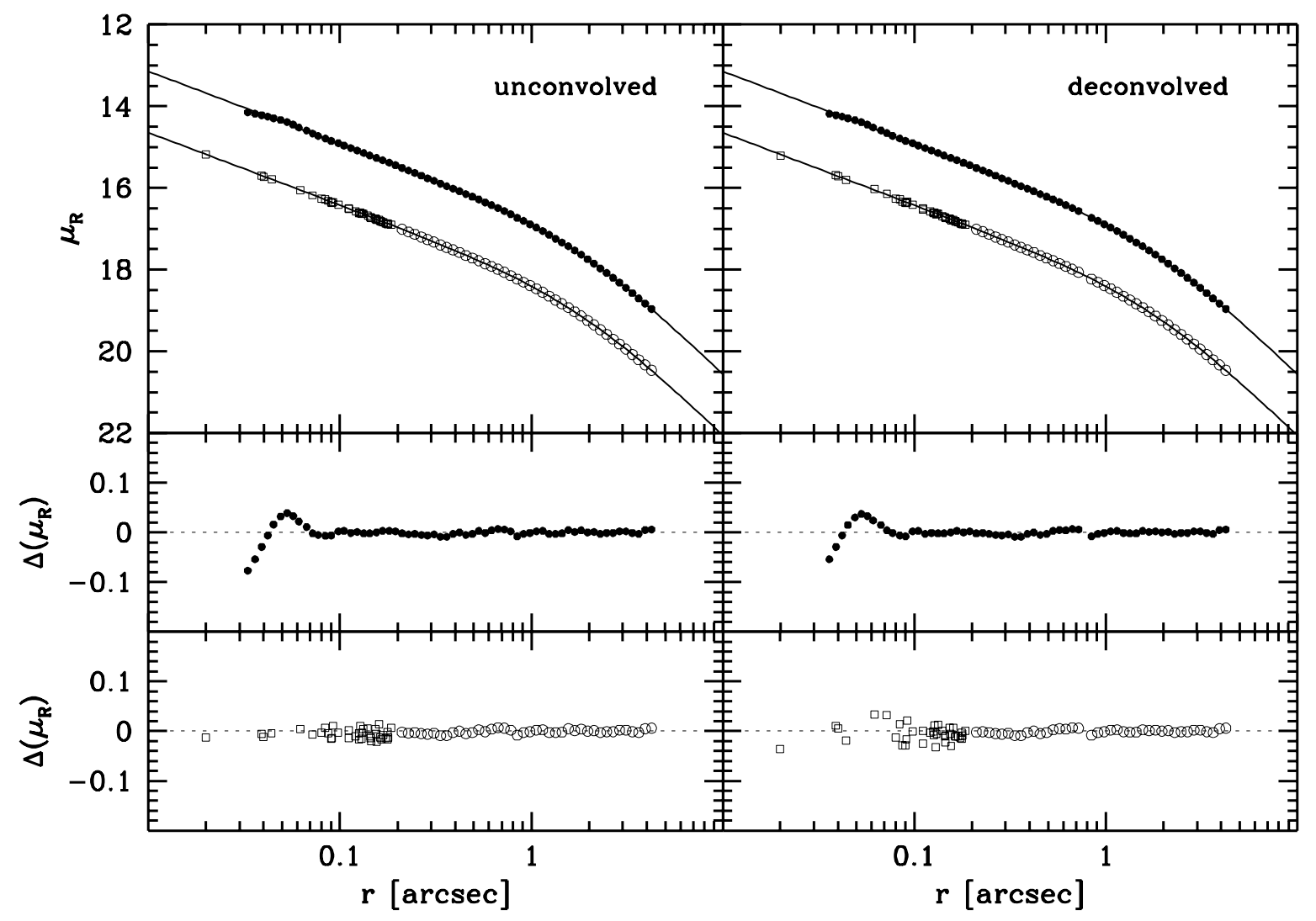

Fig. 3. - In the upper panels, the solid lines correspond to the intrinsic major axis luminosity profile of the same model as in Figure 1. Upper profiles (filled circles) correspond to the luminosity profiles determined from the isophotal analysis. Lower profiles (open symbols), offset by 1.5 magn, correspond to the luminosity profile determined with the new method utilizing the actual pixel values inside $r<0.2^{\prime \prime}$ (open squares) combined with the isophotal analysis data at $r \geq 0.2^{\prime \prime}$ (open circles). The lower panels display the residuals for the two differents methods (same symbols as in upper panels). As is evident, the new method allows us to recover the intrinsic luminosity profile with high accuracy down to very small radii, whereas luminosity profiles determined solely from the isophotal analysis result in relatively large errors at small radii. 

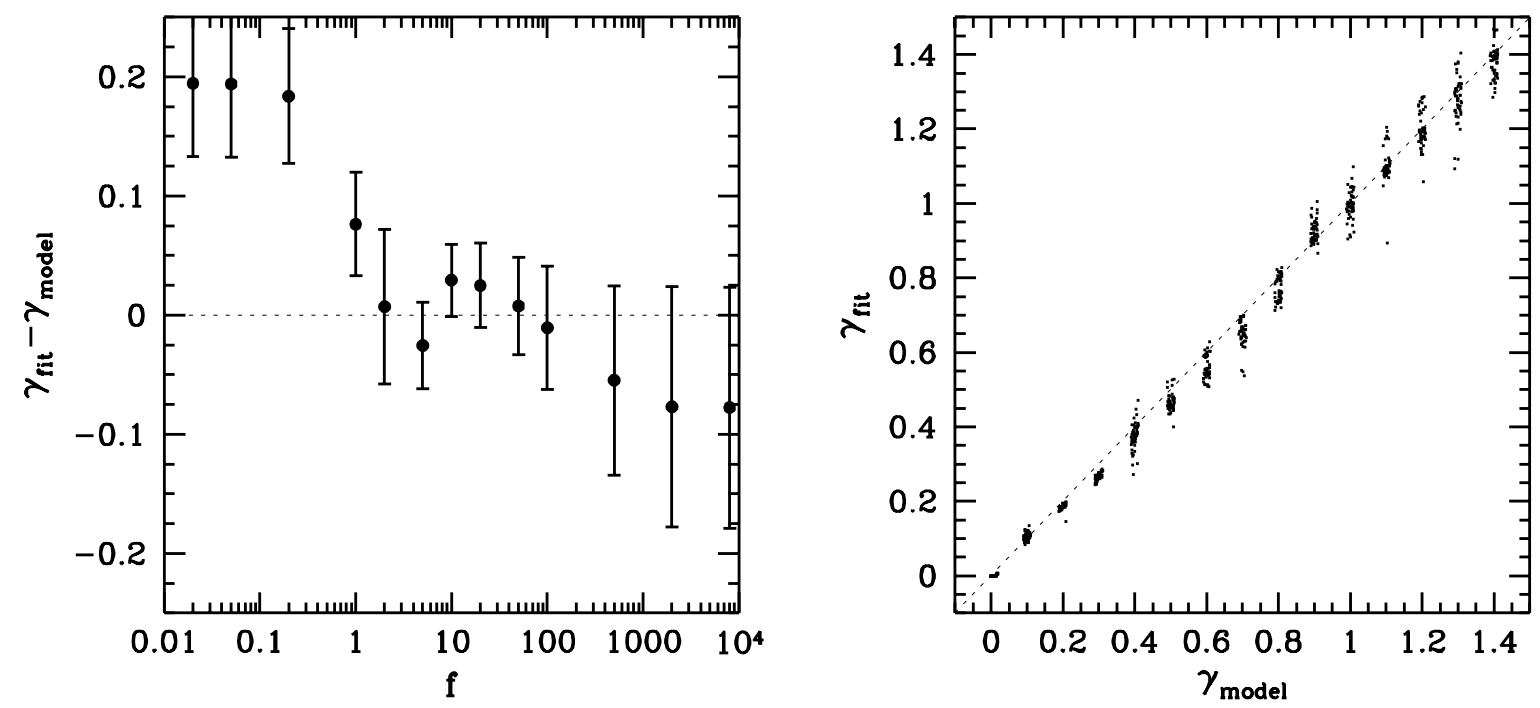

Fig. 4.- The left panel plots the average difference between the intrinsic cusp steepness $\gamma_{\text {model }}$ and the best-fit Nuker-law profile $\gamma_{\text {fit }}$ for the set of models with $\gamma=0.5$ as a function of $f$. A value of $f \simeq 5$ yields values for $\gamma_{\text {fit }}$ in good agreement with $\gamma_{\text {model }}$. For $f \lesssim 1$, too much relative weight is given to the pixel values inside $0.2^{\prime \prime}$, and $\gamma_{\text {fit }}$ is too large. For too large $f, \chi^{2}$ is completely dominated by the isophotal luminosities outside $0.2^{\prime \prime}$ and the central cusp slope becomes poorly constrained, as is evident from the large errorbars. We therefore adhere to $f=5$ for the final analysis. The right panel plots the fitted central cusp slope $\gamma_{\text {fit }}$, determined with the new fitting method as described in $\S 4.1$, versus the intrinsic slope. The dots, which correspond to a single model, are grouped in 15 sets of 50 models each. Note that $\gamma_{\text {fit }}$ is in good agreement with $\gamma_{\text {model }}$. 

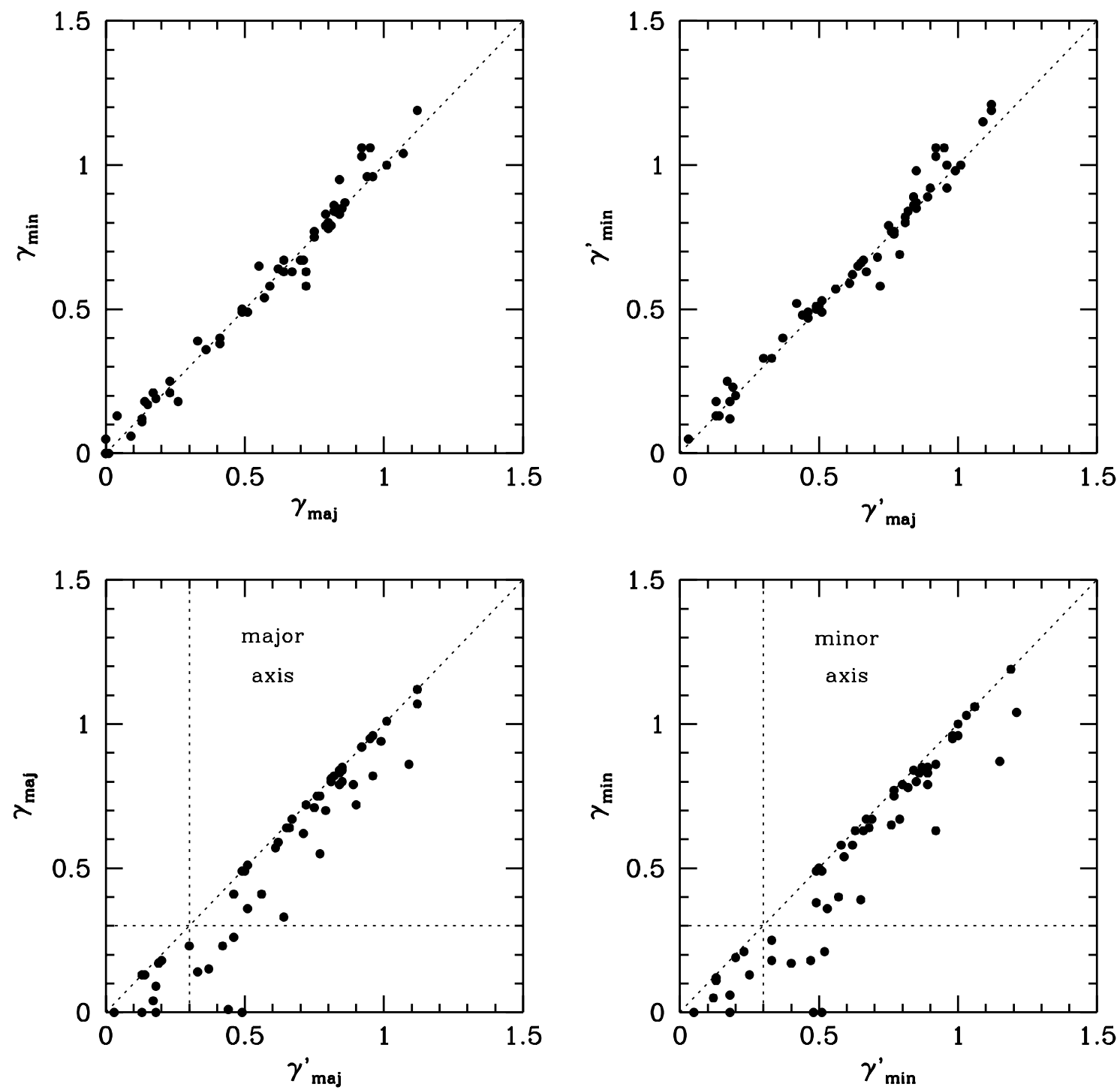

Fig. 5.- Correlations between the two different measures of the central cusp of the luminosity profile: $\gamma$ is the asymptotic logarithmic slope of the best-fit Nuker-law profile, and $\gamma^{\prime}$ is the logarithmic slope of that fit at $r=0.1^{\prime \prime}$. The indices 'maj' and 'min' refer to the major and minor axes, respectively. Both $\gamma$ and $\gamma^{\prime}$ give very similar results along both axes as shown in the upper panels. Comparing $\gamma$ to $\gamma^{\prime}$ yields a more complicated pixture. Galaxies with $\gamma>0.5$ in general have $\gamma^{\prime} \simeq \gamma$. However, for $\gamma<0.3$ about half of the galaxies have $\gamma^{\prime}<0.3$, and the other half has $\gamma^{\prime}>0.3$. See the text for a more detailed discussion. 

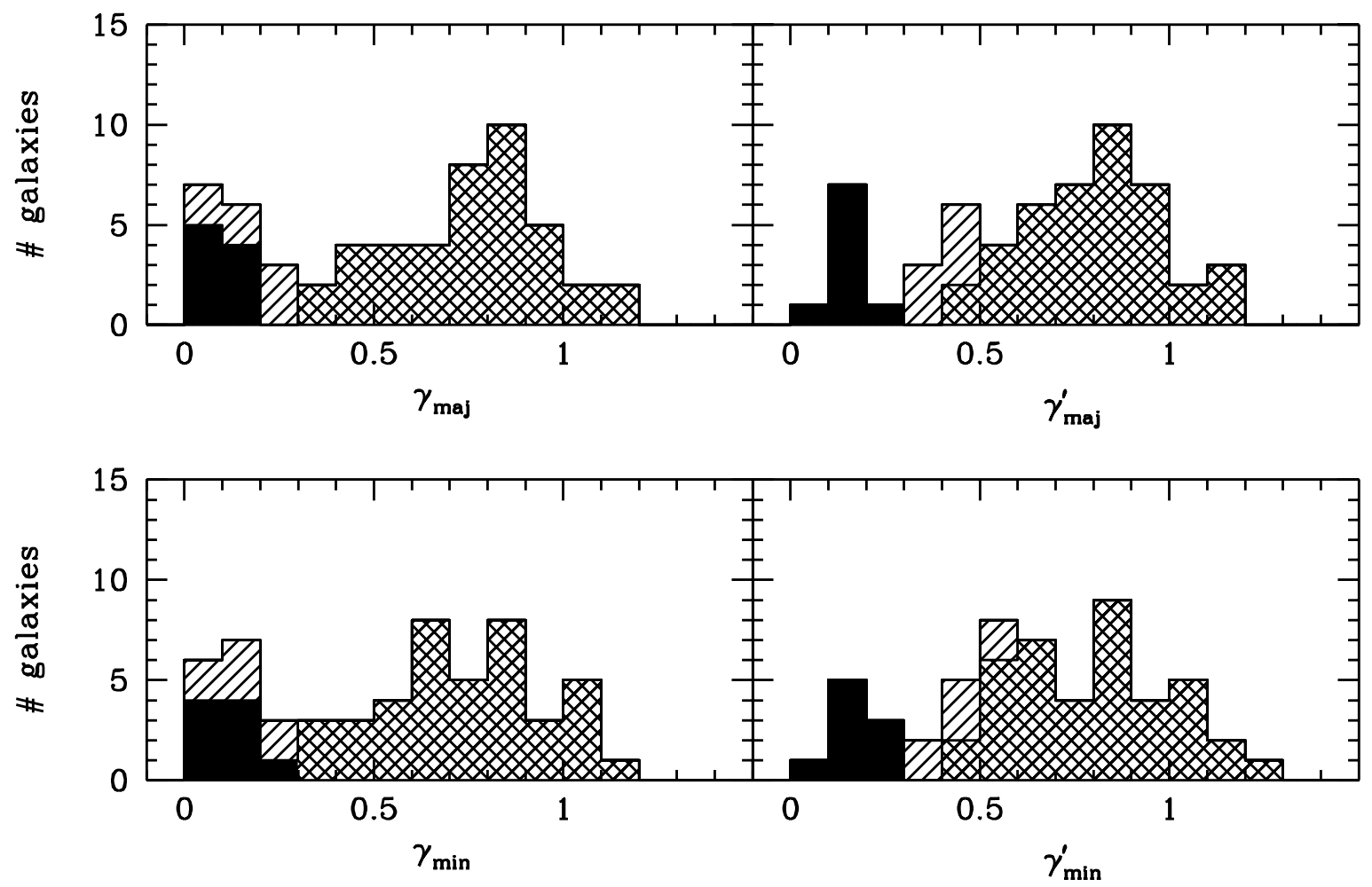

Fig. 6.- Histograms of $\gamma$ (left panels) and $\gamma^{\prime}$ (right panels) for the galaxies in our unperturbed sample. The indices 'maj' and 'min' refer to the major and minor axes, respectively. Black areas correspond to core galaxies, cross-hatched areas to power-law galaxies, and single-hatched areas to the intermediate galaxies. Although the distribution of cusp slopes reveals a clear hint for bimodality, we find less of a deficit of galaxies with $0.3 \leq \gamma \lesssim 0.5$ than F97. Note also that the intermediate galaxies themselves follow the same distribution of $\gamma$ as the core galaxies, but at the same time occupy the low- $\gamma^{\prime}$ end of the power-law galaxies. 


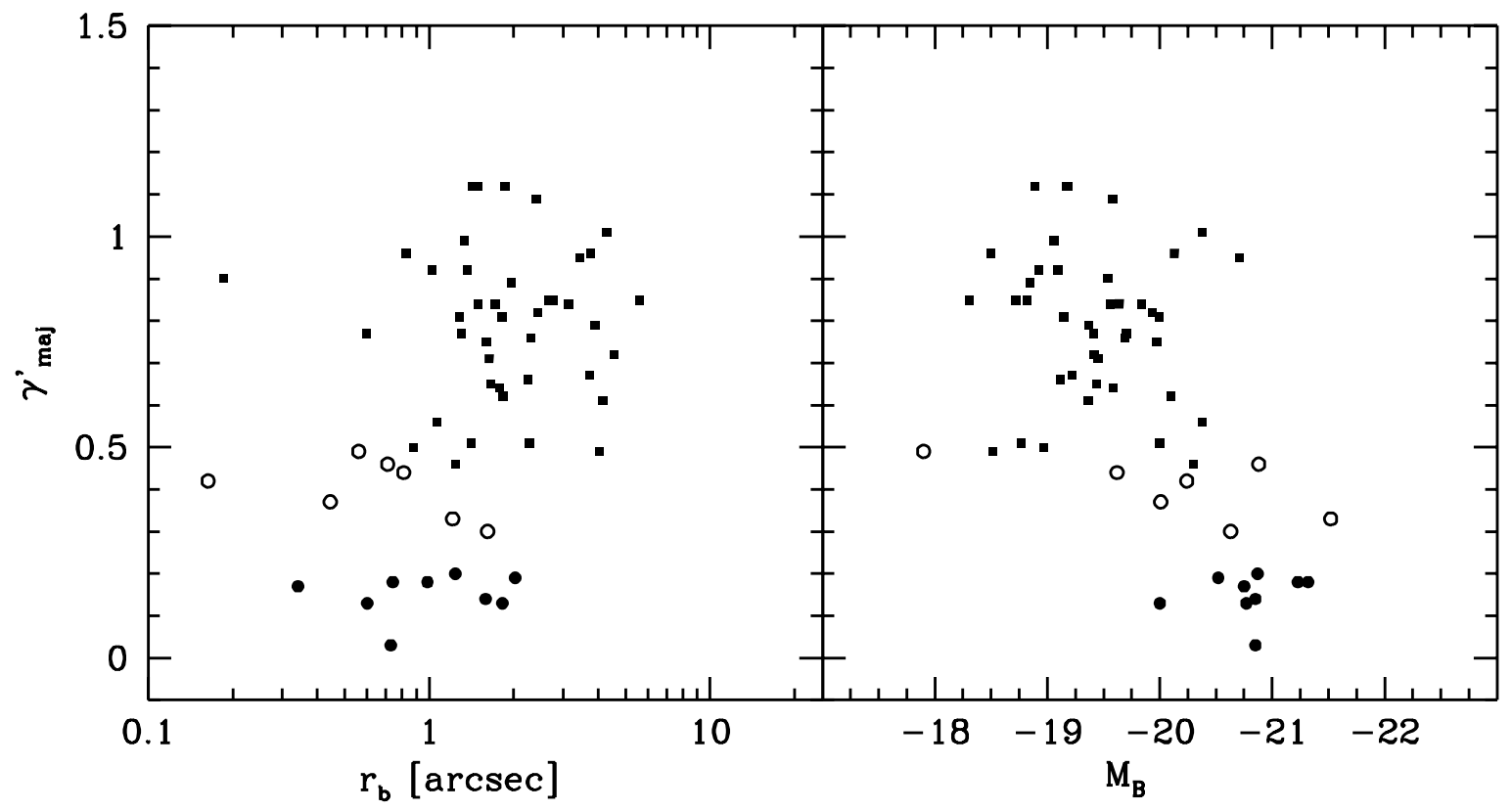

Fig. 7.- The parameter $\gamma_{\text {maj }}^{\prime}$ as function of break radius $r_{b}$ (left panel) and absolute $B$-band magnitude $M_{B}$ (right panel). Core galaxies are indicated by solid circles, intermediate galaxies by open circles, and power-law galaxies by solid squares. Note that the intermediate galaxies seem to have properties intermediate between those of core and power-law galaxies. 
Table 1. Global parameters.

\begin{tabular}{|c|c|c|c|c|c|c|c|c|c|c|}
\hline $\begin{array}{c}\text { Name } \\
(1)\end{array}$ & $\begin{array}{c}M_{B} \\
(2)\end{array}$ & $\begin{array}{l}B_{T} \\
(3)\end{array}$ & $\begin{array}{c}D \\
(4)\end{array}$ & $\begin{array}{c}\text { Type } \\
(5)\end{array}$ & $\begin{array}{l}\epsilon_{25} \\
(6)\end{array}$ & $\begin{array}{l}d_{25} \\
(7)\end{array}$ & $\begin{array}{c}V_{\text {vir }} \\
(8)\end{array}$ & $\begin{array}{c}B-V \\
\quad(9)\end{array}$ & $\begin{array}{l}f_{1.4} \\
(10)\end{array}$ & $\begin{array}{c}m_{\mathrm{FIR}} \\
(11)\end{array}$ \\
\hline ESO 378-20 & -19.58 & 13.17 & 35.6 & S0a & 0.53 & 1.134 & 3032 & 0.756 & & \\
\hline ESO 437-15 & -19.41 & 13.14 & 32.3 & S0 & 0.78 & 1.370 & 2753 & & $3.5 \pm 0.6$ & 13.46 \\
\hline ESO 443-39 & -19.41 & 13.39 & 36.3 & S0 & 0.43 & 1.146 & 3042 & & & \\
\hline ESO 447-30 & -19.84 & 12.88 & 35.0 & S0 & 0.26 & 1.233 & 2917 & & & 13.88 \\
\hline ESO 507-27 & -19.37 & 13.56 & 38.5 & S0 & 0.76 & 1.238 & 3202 & & & \\
\hline ESO 580-26 & -18.12 & 14.85 & 39.3 & S0 & 0.23 & 0.998 & 3215 & & $3.7 \pm 0.7$ & 13.15 \\
\hline IC 875 & -19.17 & 13.73 & 37.9 & S0 & 0.18 & 1.184 & 2794 & 0.731 & & \\
\hline MCG 11-14-25A & -17.90 & 15.32 & 44.2 & $\mathrm{E} / \mathrm{S} 0$ & 0.12 & 0.752 & 3304 & & & \\
\hline MCG 8-27-18 & -18.85 & 14.36 & 43.7 & $\mathrm{E} / \mathrm{S} 0$ & 0.05 & 1.002 & 3276 & & & \\
\hline NGC 2549 & -19.22 & 11.76 & 15.7 & S0 & 0.68 & 1.600 & 1064 & 0.842 & & \\
\hline NGC 2592 & -18.92 & 13.11 & 25.5 & $\mathrm{E}$ & 0.16 & 1.273 & 1987 & & & \\
\hline NGC 2634 & -19.99 & 12.50 & 31.4 & $\mathrm{E}$ & 0.00 & 1.358 & 2268 & 0.823 & & \\
\hline NGC 2699 & -18.72 & 12.97 & 21.8 & $\mathrm{E}$ & 0.06 & 1.128 & 1825 & & & \\
\hline NGC 2778 & -19.06 & 13.06 & 26.6 & $\mathrm{E}$ & 0.24 & 1.147 & 2024 & 0.850 & & \\
\hline NGC 2824 & -19.75 & 13.92 & 54.2 & S0 & 0.36 & 0.998 & 4268 & 0.784 & $9.3 \pm 0.5$ & 13.26 \\
\hline NGC 2872 & -20.38 & 12.54 & 38.3 & $\mathrm{E}$ & 0.25 & 1.279 & 3070 & 0.904 & $7.4 \pm 2.6$ & 12.09 \\
\hline NGC 2902 & -18.97 & 12.86 & 23.3 & S0 & 0.22 & 1.256 & 1991 & & & \\
\hline NGC 2950 & -19.93 & 11.52 & 19.5 & S0 & 0.32 & 1.487 & 1353 & 0.811 & & \\
\hline NGC 2986 & -20.87 & 11.27 & 26.8 & $\mathrm{E}$ & 0.12 & 1.588 & 2302 & 0.861 & & \\
\hline NGC 3065 & -19.56 & 12.69 & 28.2 & S0 & 0.05 & 1.300 & 2009 & 0.873 & $4.5 \pm 0.6$ & \\
\hline NGC 3078 & -20.71 & 11.60 & 29.0 & $\mathrm{E}$ & 0.17 & 1.512 & 2491 & 0.890 & $314 \pm 11$ & \\
\hline NGC 3193 & -19.62 & 11.64 & 17.9 & $\mathrm{E}$ & 0.08 & 1.315 & 1373 & 0.885 & & \\
\hline NGC 3226 & -19.11 & 12.08 & 17.3 & $\mathrm{E}$ & 0.12 & 1.448 & 1337 & 0.835 & $3.3 \pm 0.5$ & 10.86 \\
\hline NGC 3266 & -19.11 & 13.18 & 28.6 & S0 & 0.12 & 1.166 & 2057 & & & \\
\hline NGC 3348 & -21.23 & 11.69 & 38.5 & $\mathrm{E}$ & 0.00 & 1.354 & 2826 & 0.918 & $8.3 \pm 0.5$ & \\
\hline NGC 3377 & -18.89 & 10.90 & 9.1 & $\mathrm{E}$ & 0.39 & 1.663 & 698 & 0.801 & & \\
\hline NGC 3414 & -19.64 & 11.73 & 18.8 & S0 & 0.17 & 1.557 & 1414 & 0.898 & $4.7 \pm 0.5$ & 14.97 \\
\hline NGC 3595 & -19.69 & 12.72 & 30.4 & $\mathrm{E} / \mathrm{S} 0$ & 0.53 & 1.225 & 2248 & & & \\
\hline NGC 3613 & -20.75 & 11.49 & 28.0 & $\mathrm{E}$ & 0.45 & 1.576 & 2021 & 0.849 & & \\
\hline
\end{tabular}


Table 1-Continued

\begin{tabular}{|c|c|c|c|c|c|c|c|c|c|c|}
\hline $\begin{array}{l}\text { Name } \\
(1)\end{array}$ & $\begin{array}{c}M_{B} \\
(2)\end{array}$ & $\begin{array}{l}B_{T} \\
(3)\end{array}$ & $\begin{array}{c}D \\
(4)\end{array}$ & $\begin{array}{c}\text { Type } \\
(5)\end{array}$ & $\begin{array}{l}\epsilon_{25} \\
(6)\end{array}$ & $\begin{array}{l}d_{25} \\
(7)\end{array}$ & $\begin{array}{c}V_{\text {vir }} \\
(8)\end{array}$ & $\begin{array}{c}B-V \\
(9)\end{array}$ & $\begin{array}{l}f_{1.4} \\
(10)\end{array}$ & $\begin{array}{c}m_{\mathrm{FIR}} \\
(11)\end{array}$ \\
\hline NGC 3640 & -20.01 & 11.03 & 16.2 & $\mathrm{E}$ & 0.10 & 1.684 & 1312 & 0.853 & & \\
\hline NGC 4121 & -18.31 & 13.95 & 28.3 & $\mathrm{E}$ & 0.12 & 0.645 & 2024 & 0.824 & & \\
\hline NGC 4125 & -21.20 & 10.31 & 20.1 & $\mathrm{E}$ & 0.16 & 1.822 & 1361 & 0.850 & & 14.24 \\
\hline NGC 4128 & -19.97 & 12.58 & 32.4 & S0 & 0.65 & 1.400 & 2339 & 0.820 & & \\
\hline NGC 4168 & -20.52 & 11.83 & 29.5 & $\mathrm{E}$ & 0.18 & 1.468 & 2310 & 0.852 & $6.0 \pm 1.5$ & \\
\hline NGC 4233 & -19.71 & 12.64 & 29.6 & S0 & 0.53 & 1.374 & 2340 & 0.882 & $3.4 \pm 0.6$ & \\
\hline NGC 4291 & -20.00 & 12.04 & 25.6 & $\mathrm{E}$ & 0.14 & 1.350 & 1781 & 0.865 & & \\
\hline NGC 4365 & -20.77 & 10.21 & 15.7 & $\mathrm{E}$ & 0.24 & 1.834 & 1232 & 0.892 & & \\
\hline NGC 4474 & -19.42 & 12.13 & 20.4 & So & 0.35 & 1.404 & 1571 & 0.788 & & \\
\hline NGC 4478 & -19.36 & 11.93 & 18.1 & $\mathrm{E}$ & 0.16 & 1.273 & 1398 & 0.838 & & \\
\hline NGC 4482 & -18.52 & 13.34 & 23.6 & $\mathrm{E} / \mathrm{S} 0$ & 0.41 & 1.234 & 1843 & & & \\
\hline NGC 4494 & -20.84 & 10.41 & 17.8 & $\mathrm{E}$ & 0.05 & 1.688 & 1310 & 0.810 & & 14.83 \\
\hline NGC 4503 & -19.44 & 11.79 & 17.6 & S0a & 0.51 & 1.560 & 1360 & 0.863 & & \\
\hline NGC 4564 & -19.15 & 11.67 & 14.6 & $\mathrm{E}$ & 0.45 & 1.530 & 1116 & 0.863 & & \\
\hline NGC 4589 & -20.88 & 11.33 & 27.6 & $\mathrm{E}$ & 0.18 & 1.563 & 1945 & 0.865 & $38 \pm 2$ & 15.21 \\
\hline NGC 4621 & -18.50 & 10.41 & 6.0 & $\mathrm{E}$ & 0.24 & 1.736 & 431 & 0.879 & & \\
\hline NGC 4648 & -19.09 & 12.60 & 21.8 & $\mathrm{E}$ & 0.26 & 1.287 & 1476 & 0.829 & & \\
\hline NGC 5017 & -19.19 & 13.25 & 30.8 & $\mathrm{E}$ & 0.24 & 1.255 & 2534 & 0.882 & & \\
\hline NGC 5077 & -20.63 & 12.03 & 34.0 & $\mathrm{E}$ & 0.20 & 1.348 & 2769 & 0.942 & $161 \pm 6$ & 12.02 \\
\hline NGC 5173 & -19.52 & 13.07 & 32.8 & $\mathrm{E} / \mathrm{S} 0$ & 0.00 & 1.043 & 2419 & & $3.2 \pm 0.6$ & 16.37 \\
\hline NGC 5198 & -20.24 & 12.42 & 34.1 & $\mathrm{E} / \mathrm{S} 0$ & 0.14 & 1.334 & 2514 & 0.845 & $4.0 \pm 0.6$ & \\
\hline NGC 5283 & -18.93 & 14.13 & 40.8 & S0 & 0.10 & 1.061 & 3005 & & $13.4 \pm 0.4$ & \\
\hline NGC 5308 & -20.13 & 11.99 & 26.6 & S0a & 0.82 & 1.563 & 1877 & 0.739 & & \\
\hline NGC 5370 & -19.45 & 13.63 & 41.3 & S0 & 0.02 & 1.145 & 3053 & & & \\
\hline NGC 5557 & -21.52 & 11.62 & 42.5 & $\mathrm{E}$ & 0.21 & 1.405 & 3210 & 0.824 & & \\
\hline NGC 5576 & -20.00 & 11.41 & 19.1 & $\mathrm{E}$ & 0.23 & 1.524 & 1484 & 0.807 & & \\
\hline NGC 5796 & -20.38 & 12.43 & 36.5 & $\mathrm{E}$ & 0.25 & 1.434 & 2961 & 0.953 & $110 \pm 4$ & \\
\hline NGC 5812 & -20.10 & 11.86 & 24.6 & $\mathrm{E}$ & 0.12 & 1.420 & 1961 & 0.928 & & \\
\hline NGC 5813 & -20.85 & 11.11 & 24.6 & $\mathrm{E}$ & 0.24 & 1.663 & 1917 & 0.892 & $16 \pm 1$ & \\
\hline NGC 5831 & -19.59 & 12.06 & 21.4 & $\mathrm{E}$ & 0.13 & 1.397 & 1660 & 0.874 & & \\
\hline
\end{tabular}


Table 1-Continued

\begin{tabular}{ccccccccccc}
\hline \hline Name & $M_{B}$ & $B_{T}$ & $D$ & Type & $\epsilon_{25}$ & $d_{25}$ & $V_{\text {vir }}$ & $B-V$ & $f_{1.4}$ & $m_{\mathrm{FIR}}$ \\
$(1)$ & $(2)$ & $(3)$ & $(4)$ & $(5)$ & $(6)$ & $(7)$ & $(8)$ & $(9)$ & $(10)$ & $(11)$ \\
& & & & & & & & & & \\
\hline NGC 5846 & -21.10 & 10.74 & 23.3 & $\mathrm{E}$ & 0.06 & 1.631 & 1722 & 0.912 & $22 \pm 1$ & \\
NGC 5898 & -20.30 & 11.80 & 26.3 & $\mathrm{E}$ & 0.07 & 1.459 & 2171 & 0.929 & & \\
NGC 5903 & -20.85 & 11.58 & 30.5 & $\mathrm{E}$ & 0.26 & 1.530 & 2512 & 0.875 & $32 \pm 2$ & \\
NGC 5982 & -21.32 & 11.65 & 39.3 & $\mathrm{E}$ & 0.30 & 1.516 & 2876 & 0.816 & & \\
NGC 6278 & -19.70 & 13.15 & 37.1 & $\mathrm{~S} 0$ & 0.42 & 1.345 & 2791 & & & \\
UGC 4551 & -18.77 & 13.09 & 23.6 & $\mathrm{~S} 0$ & 0.67 & 1.322 & 1722 & 0.813 & & \\
UGC 4587 & -19.54 & 13.49 & 40.3 & $\mathrm{~S} 0$ & 0.43 & 1.215 & 3060 & & & \\
UGC 5467 & -18.87 & 13.95 & 36.7 & $\mathrm{~S} 0$ & 0.00 & 1.022 & 2894 & & $7.9 \pm 1.1$ & 13.40 \\
UGC 6062 & -18.82 & 13.74 & 32.7 & $\mathrm{~S} 0$ & 0.39 & 1.137 & 2607 & & & \\
\hline
\end{tabular}

Note. - Global parameters for the galaxies in our sample. Except for the data in the last two columns, these data are taken from the Lyon/Meudon Extragalactic Database (LEDA):

Column (1): The name of the galaxy.

Column (2): The absolute $B$-band magnitude, based on the apparent magnitudes and distances listed in columns (3) and (4), respectively.

Column (3): The total apparent $B$-band magnitude corrected for galactic extinction and redshift effects (see Paturel et al. 1997).

Column (4): Distances in Mpc, as derived from a pure Hubble expansion using the Virgo-centric infall corrected velocity (column [8]) and a Hubble constant of $H_{0}=80 \mathrm{~km} \mathrm{~s}^{-1} \mathrm{Mpc}^{-1}$.

Column (5): Morphological type, based on the code system of the Third Reference Catalogue of Bright Galaxies (de Vaucouleurs et al. 1991). A definition of this classification can be found in Paturel et al. (1997).

Column (6): The ellipticity of the $\mu_{B}=25 \mathrm{mag} \operatorname{arcsec}^{-2}$ isophote.

Column (7): The apparent diameter (in ${ }^{10} \log$ of $0.1^{\prime}$ ) of the galaxy according to the convention of the Second Reference Catalogue of Bright Galaxies (de Vaucouleurs et al. 1976).

Column (8): The radial velocity (in $\mathrm{km} \mathrm{s}^{-1}$ ) of the galaxy corrected for Virgo-centric infall (see Paturel et al. 1997 for the infall model).

Column (9): Total B-V color, corrected for galactic extinction and redshift effects.

Column (10): Radio flux (in mJy) at $1.4 \mathrm{GHz}$ from the NVSS (Condon et al. 1998).

Column (11): Far infrared magnitude, based on the IRAS $60 \mu \mathrm{m}$ and $100 \mu \mathrm{m}$ fluxes (see equation [1]). 
Table 2. Galaxy Morphology.

\begin{tabular}{|c|c|c|c|c|c|c|c|}
\hline $\begin{array}{c}\text { Name } \\
(1)\end{array}$ & $\begin{array}{l}\text { Profile } \\
\text { (2) }\end{array}$ & $\begin{array}{c}\text { Dust } \\
\text { Morph. } \\
(3)\end{array}$ & $\begin{array}{c}\text { Dust } \\
\text { Level } \\
(4)\end{array}$ & $\begin{array}{l}\text { IC } \\
(5)\end{array}$ & $\begin{array}{l}\text { Nucl. } \\
\text { (6) }\end{array}$ & $\begin{array}{l}\text { M.S. } \\
(7)\end{array}$ & $\begin{array}{l}\langle\epsilon\rangle \\
(8)\end{array}$ \\
\hline ESO 378-20 & 1 & & & $d 0 d$ & & 2 & $0.34 \pm 0.02$ \\
\hline ESO 437-15 & $?$ & $\mathrm{f}$ & III & $x d$ & & & $0.56 \pm 0.08$ \\
\hline ESO 443-39 & 1 & & & odod & & 2 & $0.25 \pm 0.04$ \\
\hline ESO 447-30 & 1 & & & odod & & 2 & $0.27 \pm 0.08$ \\
\hline ESO 507-27 & 1 & & & $d b d$ & II & & $0.49 \pm 0.07$ \\
\hline ESO 580-26 & $?$ & $\mathrm{f}$ & III & $x$ & & & $0.47 \pm 0.11$ \\
\hline IC 875 & 1 & & & $d 0$ & & & $0.55 \pm 0.05$ \\
\hline MCG 11-14-25A & ) & & & 0 & & & $0.11 \pm 0.02$ \\
\hline MCG 8-27-18 & 1 & & & 0 & & & $0.10 \pm 0.01$ \\
\hline NGC 2549 & 1 & & & $d b d$ & II & & $0.41 \pm 0.11$ \\
\hline NGC 2592 & 1 & $\mathrm{~d}$ & 0.6 & $x 0 d$ & & 1 & $0.18 \pm 0.06$ \\
\hline NGC 2634 & 1 & & & 0 & I & & $0.10 \pm 0.02$ \\
\hline NGC 2699 & 1 & d & 0.6 & $x 0 d 0$ & & 1 & $0.16 \pm 0.07$ \\
\hline NGC 2778 & 1 & & & Odo & & & $0.16 \pm 0.05$ \\
\hline NGC 2824 & $?$ & $\mathrm{f}$ & III & $x$ & & & $0.41 \pm 0.20$ \\
\hline NGC 2872 & 1 & $\mathrm{~d}$ & 0.8 & $x 0$ & & & $0.23 \pm 0.01$ \\
\hline NGC 2902 & 1 & $\mathrm{f}$ & II & $x 0$ & & & $0.05 \pm 0.02$ \\
\hline NGC 2950 & 1 & & & $d 0 d$ & & 2 & $0.29 \pm 0.04$ \\
\hline NGC 2986 & $\cap$ & & & $? 0$ & & & $0.18 \pm 0.01$ \\
\hline NGC 3065 & 1 & d & 0.4 & $x 0 d 0$ & & & $0.10 \pm 0.06$ \\
\hline NGC 3078 & 1 & d & 1.2 & $x b$ & & & $0.24 \pm 0.01$ \\
\hline NGC 3193 & ) & & & 0 & & & $0.20 \pm 0.03$ \\
\hline NGC 3226 & $?$ & $\mathrm{f}$ & III & $x$ & & & $0.17 \pm 0.04$ \\
\hline NGC 3266 & 1 & & & Odo & II & 1 & $0.08 \pm 0.08$ \\
\hline NGC 3348 & $\cap$ & & & 0 & & & $0.07 \pm 0.01$ \\
\hline NGC 3377 & 1 & $\mathrm{f}$ & I & $d$ & & & $0.53 \pm 0.01$ \\
\hline NGC 3414 & 1 & $\mathrm{f}$ & I & $x 0$ & & & $0.26 \pm 0.02$ \\
\hline NGC 3595 & 1 & & & $d b d$ & & 1 & $0.34 \pm 0.04$ \\
\hline NGC 3613 & $\cap$ & & & $d b 0$ & & & $0.24 \pm 0.06$ \\
\hline
\end{tabular}


Table 2-Continued

\begin{tabular}{|c|c|c|c|c|c|c|c|}
\hline $\begin{array}{l}\text { Name } \\
(1)\end{array}$ & $\begin{array}{c}\text { Profile } \\
\text { (2) }\end{array}$ & $\begin{array}{c}\text { Dust } \\
\text { Morph. } \\
(3)\end{array}$ & $\begin{array}{c}\text { Dust } \\
\text { Level } \\
(4)\end{array}$ & $\begin{array}{l}\mathrm{IC} \\
(5)\end{array}$ & $\begin{array}{l}\text { Nucl. } \\
\text { (6) }\end{array}$ & $\begin{array}{l}\text { M.S. } \\
(7)\end{array}$ & $\begin{array}{l}\langle\epsilon\rangle \\
(8)\end{array}$ \\
\hline NGC 3640 & ) & & & 0 & & & $0.20 \pm 0.03$ \\
\hline NGC 4121 & 1 & & & OdO & & 1 & $0.29 \pm 0.04$ \\
\hline NGC 4125 & $?$ & $\mathrm{f}$ & III & $x 0$ & & & $0.34 \pm 0.12$ \\
\hline NGC 4128 & 1 & & & $d b d$ & & & $0.39 \pm 0.02$ \\
\hline NGC 4168 & $\cap$ & $f$ & II & $x 0$ & II & & $0.18 \pm 0.03$ \\
\hline NGC 4233 & $?$ & d & 12.3 & $x$ & & & $0.30 \pm 0.18$ \\
\hline NGC 4291 & $\cap$ & & & $0 b$ & & & $0.24 \pm 0.02$ \\
\hline NGC 4365 & $\cap$ & & & $? d 0$ & & & $0.29 \pm 0.03$ \\
\hline NGC 4474 & 1 & & & $d b d$ & II & & $0.20 \pm 0.09$ \\
\hline NGC 4478 & 1 & & & $d b$ & & & $0.21 \pm 0.07$ \\
\hline NGC 4482 & 1 & & & $? 0$ & III & & $0.27 \pm 0.08$ \\
\hline NGC 4494 & $?$ & d & 1.6 & $x 0$ & & & $0.21 \pm 0.05$ \\
\hline NGC 4503 & 1 & & & $0 d 0$ & & 1 & $0.26 \pm 0.01$ \\
\hline NGC 4564 & 1 & & & od & & & $0.29 \pm 0.05$ \\
\hline NGC 4589 & ) & $\mathrm{f}$ & II & $x b$ & & & $0.19 \pm 0.03$ \\
\hline NGC 4621 & 1 & & & $d$ & & & $0.37 \pm 0.02$ \\
\hline NGC 4648 & 1 & d & 0.3 & odo & & 1 & $0.21 \pm 0.05$ \\
\hline NGC 5017 & 1 & d & 0.6 & $x 0$ & & & $0.17 \pm 0.02$ \\
\hline NGC 5077 & ) & $\mathrm{f}$ & II & $x b$ & II & & $0.29 \pm 0.03$ \\
\hline NGC 5173 & $?$ & $\mathrm{f}$ & III & $x$ & & & $0.14 \pm 0.02$ \\
\hline NGC 5198 & ) & & & 0 & & & $0.11 \pm 0.04$ \\
\hline NGC 5283 & $?$ & $\mathrm{f}$ & III & $x$ & & & $0.13 \pm 0.06$ \\
\hline NGC 5308 & 1 & & & $d 0 d$ & & & $0.47 \pm 0.02$ \\
\hline NGC 5370 & 1 & & & $0 d$ & & & $0.18 \pm 0.11$ \\
\hline NGC 5557 & ) & & & 0 & & & $0.20 \pm 0.03$ \\
\hline NGC 5576 & 1 & & & $0 b 0$ & & & $0.29 \pm 0.03$ \\
\hline NGC 5796 & $\backslash$ & $\mathrm{f}$ & I & 0 & & & $0.14 \pm 0.01$ \\
\hline NGC 5812 & 1 & d & 0.4 & $x 0$ & & & $0.02 \pm 0.01$ \\
\hline NGC 5813 & $\cap$ & $\mathrm{f}$ & II & $x 0$ & & & $0.10 \pm 0.01$ \\
\hline NGC 5831 & 1 & & & 0 & & & $0.30 \pm 0.03$ \\
\hline
\end{tabular}


Table 2-Continued

\begin{tabular}{|c|c|c|c|c|c|c|c|}
\hline $\begin{array}{c}\text { Name } \\
(1)\end{array}$ & $\begin{array}{l}\text { Profile } \\
\text { (2) }\end{array}$ & $\begin{array}{c}\text { Dust } \\
\text { Morph. } \\
(3)\end{array}$ & $\begin{array}{c}\text { Dust } \\
\text { Level } \\
(4)\end{array}$ & $\begin{array}{l}\text { IC } \\
(5)\end{array}$ & $\begin{array}{l}\text { Nucl. } \\
\text { (6) }\end{array}$ & $\begin{array}{l}\text { M.S. } \\
(7)\end{array}$ & $\begin{array}{l}\langle\epsilon\rangle \\
(8)\end{array}$ \\
\hline NGC 5846 & $?$ & $\mathrm{f}$ & III & $x 0$ & & & $0.08 \pm 0.03$ \\
\hline NGC 5898 & 1 & $\mathrm{f}$ & I & 0 & & & $0.16 \pm 0.06$ \\
\hline NGC 5903 & $\cap$ & $\mathrm{f}$ & I & $? b$ & & & $0.22 \pm 0.03$ \\
\hline NGC 5982 & $\cap$ & & & $O b$ & & & $0.31 \pm 0.07$ \\
\hline NGC 6278 & 1 & & & $b 0 d$ & & 1 & $0.22 \pm 0.09$ \\
\hline UGC 4551 & 1 & & & od & I & 1 & $0.15 \pm 0.04$ \\
\hline UGC 4587 & 1 & $\mathrm{f}$ & I & 0 & & & $0.28 \pm 0.07$ \\
\hline UGC 6062 & 1 & & & odod & & 2 & $0.30 \pm 0.04$ \\
\hline
\end{tabular}

Note. - Isophotal parameters for all the 67 galaxies in our sample. Column (2) indicates the classification where $\backslash$, ), and $\cap$ indicate power-law, intermediate and core galaxies. A question mark indicates that no meaningful luminosity profile could be determined (either due to dust or nucleation) and the galaxy classification is therefore unknown in these cases. Column (3) gives the dust morphology where 'd' stands for dust disk and ' $\mathrm{f}$ ' stands for dust filaments. Column (4) indicates either the amount of filamentary dust ranging from I (small) to III (large) or the diameter of the dust disk in arcsec, if applicable. Column (5) gives the isophotal code (IC) of the galaxy going from $r=0.2^{\prime \prime}$ to the outer region where $d, b, 0, x$, and ? indicate disky, boxy, neither disky nor boxy, undetermined due to dust, and undetermined due to small surface brightness gradient. Column (6) indicates the degree of nucleation, ranging from I (weak) to III (strong). Column (7) indicates if and how many misaligned structures (M.S.) are detected in the galaxy (see $\S$ 5.5). Column (8), finally, lists the mean ellipticity (determined over the radial interval 1.0" $<r<10.0^{\prime \prime}$ ) and its standard deviation. 
Table 3. Parameters of major axis luminosity profiles.

\begin{tabular}{|c|c|c|c|c|c|c|c|}
\hline $\begin{array}{l}\text { Name } \\
(1)\end{array}$ & $\begin{array}{c}\text { Profile } \\
(2)\end{array}$ & $\begin{array}{c}I_{b} \\
(3)\end{array}$ & $\begin{array}{c}\alpha \\
(4)\end{array}$ & $\begin{array}{c}\beta \\
(5)\end{array}$ & $\begin{array}{c}\gamma \\
(6)\end{array}$ & $\begin{array}{l}r_{b} \\
(7)\end{array}$ & $\begin{array}{l}\gamma^{\prime} \\
(8)\end{array}$ \\
\hline ESO $378-20$ & 1 & 17.61 & 0.43 & 2.00 & 0.86 & 2.41 & 1.09 \\
\hline ESO 437-15 & $?$ & & & & & & \\
\hline ESO 443-39 & 1 & 17.32 & 1.36 & 1.31 & 0.75 & 1.30 & 0.77 \\
\hline ESO 447-30 & 1 & 16.73 & 2.70 & 1.72 & 0.84 & 1.50 & 0.84 \\
\hline ESO 507-27 & 1 & 17.96 & 0.59 & 1.58 & 0.70 & 3.89 & 0.79 \\
\hline ESO 580-26 & $?$ & & & & & & \\
\hline IC 875 & $\backslash$ & 17.65 & 0.85 & 1.81 & 1.07 & 1.87 & 1.12 \\
\hline MCG 11-14-25A & ) & 16.59 & 0.69 & 2.13 & 0.00 & 0.56 & 0.49 \\
\hline MCG 8-27-18 & 1 & 18.61 & 0.80 & 1.93 & 0.79 & 1.95 & 0.89 \\
\hline NGC 2549 & $\backslash$ & 16.96 & 1.75 & 1.71 & 0.67 & 3.70 & 0.67 \\
\hline NGC 2592 & $\backslash$ & 16.61 & 3.31 & 1.60 & 0.92 & 1.37 & 0.92 \\
\hline NGC 2634 & 1 & 17.32 & 2.83 & 1.57 & 0.81 & 1.82 & 0.81 \\
\hline NGC 2699 & 1 & 17.63 & 1.66 & 1.89 & 0.84 & 2.66 & 0.85 \\
\hline NGC 2778 & 1 & 17.08 & 0.93 & 1.55 & 0.94 & 1.33 & 0.99 \\
\hline NGC 2824 & $?$ & & & & & & \\
\hline NGC 2872 & 1 & 18.11 & 2.55 & 1.66 & 1.01 & 4.27 & 1.01 \\
\hline NGC 2902 & 1 & 16.34 & 1.93 & 1.57 & 0.49 & 0.88 & 0.50 \\
\hline NGC 2950 & 1 & 16.16 & 2.40 & 1.81 & 0.82 & 2.43 & 0.82 \\
\hline NGC 2986 & $\cap$ & 16.09 & 1.77 & 1.50 & 0.18 & 1.24 & 0.20 \\
\hline NGC 3065 & 1 & 17.97 & 0.99 & 2.28 & 0.79 & 3.14 & 0.84 \\
\hline NGC 3078 & 1 & 17.19 & 2.35 & 1.60 & 0.95 & 3.43 & 0.95 \\
\hline NGC 3193 & ) & 15.46 & 0.59 & 1.89 & 0.01 & 0.81 & 0.44 \\
\hline NGC 3226 & $?$ & & & & & & \\
\hline NGC 3266 & 1 & 17.78 & 1.34 & 2.06 & 0.64 & 2.25 & 0.66 \\
\hline NGC 3348 & $\cap$ & 16.00 & 1.17 & 1.53 & 0.09 & 0.99 & 0.18 \\
\hline NGC 3377 & 1 & 15.37 & 3.15 & 1.35 & 1.12 & 1.42 & 1.12 \\
\hline NGC 3414 & 1 & 16.46 & 1.40 & 1.45 & 0.83 & 1.72 & 0.84 \\
\hline NGC 3595 & 1 & 17.43 & 2.33 & 1.52 & 0.75 & 2.30 & 0.76 \\
\hline NGC 3613 & $\cap$ & 15.11 & 1.53 & 1.06 & 0.04 & 0.34 & 0.17 \\
\hline
\end{tabular}


Table 3-Continued

\begin{tabular}{|c|c|c|c|c|c|c|c|}
\hline $\begin{array}{c}\text { Name } \\
(1)\end{array}$ & $\begin{array}{c}\text { Profile } \\
\quad(2)\end{array}$ & $\begin{array}{l}I_{b} \\
(3)\end{array}$ & $\begin{array}{c}\alpha \\
(4)\end{array}$ & $\begin{array}{c}\beta \\
(5)\end{array}$ & $\begin{array}{c}\gamma \\
(6)\end{array}$ & $\begin{array}{c}r_{b} \\
(7)\end{array}$ & $\begin{array}{c}\gamma^{\prime} \\
(8)\end{array}$ \\
\hline NGC 3640 & ) & 15.24 & 0.87 & 1.16 & 0.15 & 0.44 & 0.37 \\
\hline NGC 4121 & 1 & 19.69 & 1.51 & 3.65 & 0.85 & 5.60 & 0.85 \\
\hline NGC 4125 & $?$ & & & & & & \\
\hline NGC 4128 & 1 & 16.31 & 1.13 & 1.69 & 0.71 & 1.59 & 0.75 \\
\hline NGC 4168 & $\cap$ & 17.45 & 1.43 & 1.39 & 0.17 & 2.02 & 0.19 \\
\hline NGC 4233 & $?$ & & & & & & \\
\hline NGC 4291 & $\cap$ & 15.06 & 1.35 & 1.62 & 0.00 & 0.60 & 0.13 \\
\hline NGC 4365 & $\cap$ & 16.08 & 2.29 & 1.28 & 0.13 & 1.82 & 0.13 \\
\hline NGC 4474 & 1 & 17.97 & 2.28 & 1.65 & 0.72 & 4.55 & 0.72 \\
\hline NGC 4478 & 1 & 17.57 & 0.93 & 1.73 & 0.57 & 4.16 & 0.61 \\
\hline NGC 4482 & 1 & 19.63 & 3.43 & 1.01 & 0.49 & 4.04 & 0.49 \\
\hline NGC 4494 & $?$ & 15.21 & & 1.25 & & & \\
\hline NGC 4503 & 1 & 16.53 & 1.77 & 1.30 & 0.64 & 1.65 & 0.65 \\
\hline NGC 4564 & 1 & 15.89 & 1.43 & 1.27 & 0.80 & 1.28 & 0.81 \\
\hline NGC 4589 & ) & 15.64 & 0.82 & 1.44 & 0.26 & 0.71 & 0.46 \\
\hline NGC 4621 & 1 & 16.57 & 2.53 & 1.40 & 0.96 & 3.75 & 0.96 \\
\hline NGC 4648 & 1 & 15.87 & 3.73 & 1.54 & 0.92 & 1.02 & 0.92 \\
\hline NGC 5017 & 1 & 16.92 & 2.97 & 1.59 & 1.12 & 1.48 & 1.12 \\
\hline NGC 5077 & ) & 16.49 & 1.09 & 1.67 & 0.23 & 1.61 & 0.30 \\
\hline NGC 5173 & $?$ & & & & & & \\
\hline NGC 5198 & ) & 14.79 & 2.61 & 1.13 & 0.23 & 0.16 & 0.42 \\
\hline NGC 5283 & $?$ & & & & & & \\
\hline NGC 5308 & 1 & 15.55 & 0.39 & 1.27 & 0.82 & 0.83 & 0.96 \\
\hline NGC 5370 & 1 & 17.84 & 0.78 & 1.50 & 0.62 & 1.63 & 0.71 \\
\hline NGC 5557 & ) & 16.18 & 0.80 & 1.77 & 0.14 & 1.21 & 0.33 \\
\hline NGC 5576 & 1 & 15.79 & 0.79 & 1.73 & 0.36 & 1.41 & 0.51 \\
\hline NGC 5796 & 1 & 16.19 & 0.83 & 1.67 & 0.41 & 1.06 & 0.56 \\
\hline NGC 5812 & 1 & 16.61 & 1.25 & 1.67 & 0.59 & 1.84 & 0.62 \\
\hline NGC 5813 & $\cap$ & 15.77 & 1.90 & 1.33 & 0.00 & 0.73 & 0.03 \\
\hline NGC 5831 & 1 & 16.90 & 0.47 & 1.84 & 0.33 & 1.78 & 0.64 \\
\hline
\end{tabular}


Table 3 - Continued

\begin{tabular}{cccccccc}
\hline \hline Name & Profile & $I_{b}$ & $\alpha$ & $\beta$ & $\gamma$ & $r_{b}$ & $\gamma^{\prime}$ \\
$(1)$ & $(2)$ & $(3)$ & $(4)$ & $(5)$ & $(6)$ & $(7)$ & $(8)$ \\
& & & & & & & \\
\hline NGC 5846 & $?$ & & & & & & \\
NGC 5898 & $\backslash$ & 16.32 & 1.23 & 1.57 & 0.41 & 1.24 & 0.46 \\
NGC 5903 & $\cap$ & 16.84 & 1.80 & 1.48 & 0.13 & 1.59 & 0.14 \\
NGC 5982 & $\cap$ & 15.60 & 0.99 & 1.52 & 0.00 & 0.74 & 0.18 \\
NGC 6278 & $\backslash$ & 15.69 & 0.76 & 1.62 & 0.55 & 0.60 & 0.77 \\
UGC 4551 & $\backslash$ & 17.06 & 2.19 & 2.16 & 0.51 & 2.26 & 0.51 \\
UGC 4587 & $\backslash$ & 15.48 & 1.04 & 1.24 & 0.72 & 0.19 & 0.90 \\
UGC 6062 & $\backslash$ & 18.35 & 0.90 & 1.81 & 0.80 & 2.75 & 0.85 \\
\hline
\end{tabular}

Note. - Parameterizations of the major axis luminosity profiles. Columns (2) indicates the classification of the galaxy (copied from column (2) in Table 2). Columns (3) - (7) list the best fit parameters of the Nuker-law (equation [3]). Here the break radius, $r_{b}$, is in $\operatorname{arcsec}$ and $I_{b}$ in mag $\operatorname{arcsec}^{-2}$. Column (8) lists the value of $\gamma^{\prime}$ (see $\S 5.2$ for definition). 
Table 4. Parameters of minor axis luminosity profiles.

\begin{tabular}{|c|c|c|c|c|c|c|c|}
\hline $\begin{array}{l}\text { Name } \\
(1)\end{array}$ & $\begin{array}{c}\text { Profile } \\
(2)\end{array}$ & $\begin{array}{c}I_{b} \\
(3)\end{array}$ & $\begin{array}{c}\alpha \\
(4)\end{array}$ & $\begin{array}{l}\beta \\
(5)\end{array}$ & $\begin{array}{c}\gamma \\
(6)\end{array}$ & $\begin{array}{l}r_{b} \\
(7)\end{array}$ & $\begin{array}{l}\gamma^{\prime} \\
(8)\end{array}$ \\
\hline ESO 378-20 & 1 & 17.96 & 0.39 & 2.06 & 0.87 & 1.98 & 1.15 \\
\hline ESO 437-15 & $?$ & & & & & & \\
\hline ESO 443-39 & $\backslash$ & 17.52 & 1.53 & 1.63 & 0.75 & 1.19 & 0.77 \\
\hline ESO 447-30 & 1 & 16.88 & 1.31 & 1.60 & 0.83 & 1.19 & 0.86 \\
\hline ESO 507-27 & 1 & 17.71 & 1.56 & 2.25 & 0.67 & 1.66 & 0.69 \\
\hline ESO 580-26 & $?$ & & & & & & \\
\hline IC 875 & $\backslash$ & 18.93 & 0.41 & 1.78 & 1.04 & 2.14 & 1.21 \\
\hline MCG 11-14-25A & ) & 16.69 & 0.70 & 2.21 & 0.00 & 0.56 & 0.51 \\
\hline MCG 8-27-18 & 1 & 18.39 & 0.82 & 1.87 & 0.79 & 1.53 & 0.89 \\
\hline NGC 2549 & $\backslash$ & 15.88 & 3.49 & 1.55 & 0.63 & 0.94 & 0.63 \\
\hline NGC 2592 & $\backslash$ & 17.40 & 2.40 & 1.86 & 1.06 & 1.92 & 1.06 \\
\hline NGC 2634 & 1 & 17.33 & 2.56 & 1.64 & 0.79 & 1.66 & 0.80 \\
\hline NGC 2699 & 1 & 17.87 & 1.06 & 1.84 & 0.95 & 2.40 & 0.98 \\
\hline NGC 2778 & $\backslash$ & 16.56 & 1.49 & 1.53 & 0.96 & 0.77 & 0.98 \\
\hline NGC 2824 & $?$ & & & & & & \\
\hline NGC 2872 & 1 & 17.84 & 3.12 & 1.58 & 1.00 & 2.76 & 1.00 \\
\hline NGC 2902 & 1 & 16.27 & 2.14 & 1.50 & 0.49 & 0.77 & 0.51 \\
\hline NGC 2950 & 1 & 16.16 & 1.66 & 1.78 & 0.84 & 1.65 & 0.84 \\
\hline NGC 2986 & $\cap$ & 16.05 & 1.86 & 1.45 & 0.19 & 0.97 & 0.20 \\
\hline NGC 3065 & 1 & 18.04 & 0.86 & 2.17 & 0.83 & 2.97 & 0.89 \\
\hline NGC 3078 & 1 & 17.49 & 3.42 & 1.66 & 1.06 & 3.26 & 1.06 \\
\hline NGC 3193 & ) & 14.21 & 1.18 & 1.18 & 0.00 & 0.14 & 0.48 \\
\hline NGC 3226 & $?$ & & & & & & \\
\hline NGC 3266 & 1 & 17.28 & 2.39 & 1.91 & 0.67 & 1.46 & 0.67 \\
\hline NGC 3348 & $\cap$ & 16.06 & 1.08 & 1.61 & 0.06 & 0.98 & 0.18 \\
\hline NGC 3377 & 1 & 15.91 & & 1.29 & & & \\
\hline NGC 3414 & 1 & 16.29 & 1.13 & 1.39 & 0.85 & 1.10 & 0.89 \\
\hline NGC 3595 & 1 & 17.26 & 2.43 & 1.75 & 0.77 & 1.34 & 0.77 \\
\hline NGC 3613 & $\cap$ & 15.73 & 1.23 & 1.46 & 0.13 & 0.65 & 0.25 \\
\hline
\end{tabular}


Table 4-Continued

\begin{tabular}{|c|c|c|c|c|c|c|c|}
\hline $\begin{array}{c}\text { Name } \\
(1)\end{array}$ & $\begin{array}{c}\text { Profile } \\
\quad(2)\end{array}$ & $\begin{array}{l}I_{b} \\
(3)\end{array}$ & $\begin{array}{c}\alpha \\
(4)\end{array}$ & $\begin{array}{c}\beta \\
(5)\end{array}$ & $\begin{array}{c}\gamma \\
(6)\end{array}$ & $\begin{array}{c}r_{b} \\
(7)\end{array}$ & $\begin{array}{c}\gamma^{\prime} \\
(8)\end{array}$ \\
\hline NGC 3640 & ) & 15.41 & 0.86 & 1.28 & 0.17 & 0.48 & 0.40 \\
\hline NGC 4121 & 1 & 19.32 & 1.28 & 2.90 & 0.85 & 3.48 & 0.87 \\
\hline NGC 4125 & $?$ & & & & & & \\
\hline NGC 4128 & 1 & 17.30 & 0.83 & 2.22 & 0.67 & 1.98 & 0.79 \\
\hline NGC 4168 & $\cap$ & 17.48 & 1.43 & 1.30 & 0.21 & 1.64 & 0.23 \\
\hline NGC 4233 & $?$ & & & & & & \\
\hline NGC 4291 & $\cap$ & 15.07 & 1.36 & 1.65 & 0.00 & 0.47 & 0.18 \\
\hline NGC 4365 & $\cap$ & 16.25 & 1.54 & 1.39 & 0.11 & 1.55 & 0.13 \\
\hline NGC 4474 & 1 & 17.43 & 3.83 & 1.95 & 0.58 & 2.38 & 0.58 \\
\hline NGC 4478 & 1 & 17.63 & 0.87 & 1.61 & 0.54 & 3.31 & 0.59 \\
\hline NGC 4482 & 1 & 19.92 & 2.17 & 1.50 & 0.50 & 4.02 & 0.50 \\
\hline NGC 4494 & $?$ & 15.58 & & 1.18 & & & \\
\hline NGC 4503 & 1 & 16.89 & 1.21 & 1.60 & 0.63 & 1.70 & 0.66 \\
\hline NGC 4564 & 1 & 16.90 & 1.13 & 1.93 & 0.78 & 2.10 & 0.82 \\
\hline NGC 4589 & ) & 15.64 & 0.71 & 1.45 & 0.18 & 0.54 & 0.47 \\
\hline NGC 4621 & 1 & 17.52 & 0.69 & 1.49 & 0.96 & 4.81 & 1.00 \\
\hline NGC 4648 & 1 & 16.50 & 3.16 & 1.75 & 1.03 & 1.29 & 1.03 \\
\hline NGC 5017 & 1 & 17.75 & 1.81 & 1.68 & 1.19 & 2.12 & 1.19 \\
\hline NGC 5077 & ) & 16.48 & 1.15 & 1.74 & 0.25 & 1.19 & 0.33 \\
\hline NGC 5173 & $?$ & & & & & & \\
\hline NGC 5198 & ) & 14.81 & 2.04 & 1.15 & 0.21 & 0.14 & 0.52 \\
\hline NGC 5283 & $?$ & & & & & & \\
\hline NGC 5308 & 1 & 18.39 & 0.85 & 2.18 & 0.86 & 3.95 & 0.92 \\
\hline NGC 5370 & 1 & 18.61 & 1.26 & 2.51 & 0.64 & 2.40 & 0.68 \\
\hline NGC 5557 & ) & 15.74 & 1.11 & 1.54 & 0.18 & 0.67 & 0.33 \\
\hline NGC 5576 & 1 & 15.57 & 0.91 & 1.76 & 0.36 & 0.89 & 0.53 \\
\hline NGC 5796 & 1 & 16.35 & 0.82 & 1.71 & 0.40 & 1.05 & 0.57 \\
\hline NGC 5812 & 1 & 16.69 & 1.22 & 1.73 & 0.58 & 1.91 & 0.62 \\
\hline NGC 5813 & $\cap$ & 15.88 & 1.61 & 1.41 & 0.00 & 0.74 & 0.05 \\
\hline NGC 5831 & 1 & 15.16 & 0.99 & 1.34 & 0.39 & 0.27 & 0.65 \\
\hline
\end{tabular}


Table 4-Continued

\begin{tabular}{cccccccc}
\hline \hline Name & Profile & $I_{b}$ & $\alpha$ & $\beta$ & $\gamma$ & $r_{b}$ & $\gamma^{\prime}$ \\
$(1)$ & $(2)$ & $(3)$ & $(4)$ & $(5)$ & $(6)$ & $(7)$ & $(8)$ \\
& & & & & & \\
\hline NGC 5846 & $?$ & & & & & & \\
NGC 5898 & $\backslash$ & 16.63 & 0.90 & 1.61 & 0.38 & 1.35 & 0.49 \\
NGC 5903 & $\cap$ & 16.66 & 2.30 & 1.35 & 0.12 & 0.98 & 0.13 \\
NGC 5982 & $\cap$ & 15.26 & 1.91 & 1.42 & 0.05 & 0.46 & 0.12 \\
NGC 6278 & $\backslash$ & 16.31 & 1.11 & 1.96 & 0.65 & 0.86 & 0.76 \\
UGC 4551 & $\backslash$ & 17.39 & 1.95 & 2.57 & 0.49 & 2.48 & 0.49 \\
UGC 4587 & $\backslash$ & 15.97 & 0.72 & 1.47 & 0.63 & 0.23 & 0.92 \\
UGC 6062 & $\backslash$ & 18.46 & 1.05 & 2.05 & 0.80 & 2.16 & 0.85 \\
\hline
\end{tabular}

Note. - Same as Table 3, except now for the minor axis luminosity profiles.

Table 5 .

\begin{tabular}{l|rrrrr}
\hline \hline \multicolumn{1}{c|}{ Profile } & $\mathrm{d}$ & $\mathrm{db}$ & $\mathrm{b}$ & \multicolumn{1}{c}{0} & Total \\
& & & & & \\
\hline Power-law & 20 & 7 & 2 & 12 & 41 \\
Intermediate & 0 & 0 & 2 & 5 & 7 \\
Core & 1 & 1 & 3 & 4 & 9 \\
\hline Total & 21 & 8 & 7 & 21 & 57 \\
\hline
\end{tabular}

Note. - Correlation of luminosity profiles with isophotal structure. For each class of luminosity profiles (power-law, intermediate, and core) we indicate the number of galaxies (in the unperturbed sample) with a common isophotal characteristic. We distinguish between disky 'd', boxy 'b', diskyboxy 'db', and regular ' 0 ' (see $\S 5.4 .1$ for definitions). Note that most disky and disky-boxy galaxies are power-law galaxies, whereas most boxy galaxies are classified as either core or intermediate galaxies 